\title{
FINITE-DIMENSIONAL APPROXIMATION FOR OPTIMAL FIXED-ORDER COMPENSATION OF DISTRIBUTED PARAMETER SYSTEMS
}

\author{
DENNIS S. BERNSTEIN \\ Government Aerospace Systems Division, Harris Corporation, MS 22/4848, Melbourne, FL 32902, U.S.A. \\ I. GARY ROSEN \\ Department of Mathematics, University of Southern California, Los Angeles, CA 90089, U.S.A.
}

\begin{abstract}
SUMMARY
In controlling distributed parameter systems it is often desirable to obtain low-order, finite-dimensional controllers in order to minimize real-time computational requirements. Standard approaches to this problem employ model/controller reduction techniques in conjunction with LQG theory. In this paper we consider the finite-dimensional approximation of the infinite-dimensional Bernstein/Hyland optimal projection theory. Our approach yields fixed-finite-order controllers which are optimal with respect to high-order, approximating, finite-dimensional plant models. We illustrate the technique by computing a sequence of first-order controllers for one-dimensional, single-input/single-output parabolic (heat/ diffusion) and hereditary systems using a spline-based, Ritz-Galerkin, finite element approximation. Our numerical studies indicate convergence of the feedback gains with less than $2 \%$ performance degradation over full-order LQG controllers for the parabolic system and 10\% degradation for the hereditary system.
\end{abstract}

KEY WORDS Finite-dimensional compensation Distributed parameter systems Optimal control

\section{INTRODUCTION}

Approximation methods for the optimal control of distributed parameter systems have been widely studied. In particular, the approach taken in References 1-12 involves approximating the original distributed parameter system by a sequence of finite-dimensional systems and then using finite-dimensional control design techniques to obtain a sequence of approximating, suboptimal control laws, observers or compensators. Furthermore, in these treatments it was demonstrated that if the open-loop system is approximated appropriately, then it is possible to guarantee convergence of the sequence of suboptimal controllers, observers or compensators respectively to the optimal controller, observer or compensator for the original infinitedimensional system. In addition, it can be shown that when the approximating suboptimal control laws or estimators are applied to the original system, near-optimal performance can frequently be obtained. These ideas have been pursued in the context of both open- and closedloop control, in both continuous and discrete time, and for both full-state-feedback control and LQG (i.e. Kalman-filter-based) state estimation and compensation.

In practical situations, however, it is often of interest to obtain the simplest (i.e. the lowestorder) controller which provides a given desired feedback performance. This is usually achieved in one of two ways: either the plant approximation order is reduced prior to controller design or reduction techniques are applied to a given high-order control law. Unfortunately, the 
former approach may result in undesirable spillover effects while the latter may yield low-order controllers of low authority which perform unacceptably. In fact, with the second approach this may occur even when a suitable controller is known to exist. For example, as is shown in Reference 13, controller reduction techniques may even destabilize the closed-loop system.

A third, more direct approach involves fixing the controller order a priori and then optimizing a performance criterion over the class of fixed-order controllers. In a finitedimensional setting a set of necessary conditions in the form of four coupled matrix equations (as a direct extension of the pair of separated Riccati equations of LQG theory) which characterize the optimal fixed-order compensator was derived in Reference 14. These four equations are coupled via an oblique projection (idempotent) matrix. In the full-order case this projection becomes the identity, thus effectively eliminating the additional two equations, and the necessary conditions reduce to the standard LQG Riccati equations.

The notion that this direct (i.e. fixed-finite-order) approach can be applied to distributed parameter systems was first suggested by Johnson ${ }^{15}$ and further developed in References 16 and 17. To realize such an approach, however, would require a suitable generalization of the optimality conditions for the finite-dimensional fixed-order theory. This result was subsequently obtained in Reference 18, where the matrix optimal projection equations obtained in Reference 14 for finite-dimensional systems were extended to a set of four coupled operator Riccati and Lyapunov equations characterizing optimal fixed-finite-order controllers for infinite-dimensional systems.

In developing numerical schemes to actually compute fixed-finite-order compensators for infinite-dimensional systems, one might consider an approach wherein LQG reduction procedures are applied to a sequence of controllers obtained by using finite-dimensional fullorder design techniques in conjunction with high-order finite-dimensional plant approximations. However, such an approach is unappealing for two reasons. First, since such methods are not predicated on the minimization of a performance index, prospects for convergence are slim. Secondly, controller reduction methods have not proven to be reliable in producing stabilizing compensators (see e.g. Reference 13).

Hence, as an alternative, we develop an abstract approximation framework (and ultimately computational schemes) which combines the infinite-dimensional optimal projection theory of Reference 18 with the approximation ideas developed in References 9-12 for infinitedimensional LQG problems. More precisely, our approach involves constructing a sequence of approximating finite-dimensional subspaces of the original, underlying, infinite-dimensional Hilbert state space along with corresponding sequences of bounded linear operators which approximate the given input, output and system operators. Then, by choosing bases for these approximating subspaces and applying the finite-dimensional optimal projection theory, a sequence of matrix equations characterizing a sequence of approximating optimal fixed-finiteorder compensators for the distributed system is obtained. Finally, numerical techniques for solving the matrix optimal projection equations (e.g. the homotopic continuation algorithm described in References 19 and 20) can be used to compute the sequence of approximating gains.

Our primary aim in this paper is to describe the general approach we are proposing, to discuss its implementation and to demonstrate its feasibility and practicality. We offer no convergence arguments here but rather hope to treat them in a more theoretical paper to follow. Instead we consider the application of our technique to two examples. One involves the control of a one-dimensional, single-input/single-output parabolic (heat/diffusion) system while the other involves a single-input/single-output one-dimensional hereditary control system. These relatively simple examples have been used throughout the distributed parameter control 
literature to illustrate the application of new theories and techniques. A detailed discussion of the application of our ideas to more complex control systems, e.g. the vibration control of flexible structures, will also appear elsewhere. We use spline-based Ritz-Galerkin finite element schemes to approximate the open-loop systems (one for which convergence can be demonstrated in the LQG case) and present and discuss some of the numerical results which we have obtained using our general approximation framework.

We now outline the remainder of the paper. In Section 2 we briefly review the infinitedimensional optimal projection theory from Reference 18, describe the approximation framework and derive the corresponding equivalent matrix equations and feedback gains which characterize the approximating fixed-finite-order compensator. In Section 3 we consider the examples, construct the approximation schemes and discuss our numerical findings. Section 4 contains a summary and some concluding remarks.

\section{OPTIMAL PROJECTION THEORY AND FINITE-DIMENSIONAL APPROXIMATION}

We consider the following fixed-finite-order dynamic compensation problem. Given the infinite-dimensional control system

$$
\dot{x}(t)=A x(t)+B u(t)+H_{1} w(t)
$$

with measurements

$$
y(t)=C x(t)+H_{2} w(t)
$$

where $t \in[0, \infty)$, design a finite-dimensional, $n_{\mathrm{c}}$ th-order dynamic compensator

$$
\begin{gathered}
\dot{x}_{\mathrm{c}}(t)=A_{\mathrm{c}} x_{\mathrm{c}}(t)+B_{\mathrm{c}} y(t) \\
u(t)=C_{\mathrm{c}} x_{\mathrm{c}}(t)
\end{gathered}
$$

which minimizes the steady-state performance criterion

$$
J\left(A_{\mathrm{c}}, B_{\mathrm{c}}, C_{\mathrm{c}}\right) \triangleq \lim _{t \rightarrow \infty} \mathbb{E}\left[\left\langle R_{1} x(t), x(t)\right\rangle+u(t)^{\mathrm{T}} R_{2} u(t)\right]
$$

For convenience we denote the infinite-dimensional plant by $\Pi$; that is,

$$
\Pi \triangleq\left\{A, B, C, R_{1}, R_{2}, V_{1}, V_{2}\right\}
$$

Here $x(t)$ lies in a real, separable Hilbert space $\mathscr{X}$ with inner product $\langle\cdot, \cdot\rangle, A$ : $\operatorname{Dom}(A) \subset \mathscr{X} \rightarrow \mathscr{X}$ is a closed, densely defined operator which generates a $C_{0}$ semigroup $\{T(t): t \geqslant 0\}$ of bounded linear operators on $\mathscr{X}, B \in \mathscr{L}\left(\mathbb{R}^{m}, \mathscr{X}\right)$ and $C \in \mathscr{L}\left(\mathscr{X}, \mathbb{R}^{l}\right)$. We assume that the state and measurement are corrupted by a white noise signal $w(t)$ in a real, separable Hilbert space $\hat{\mathscr{X}}$ (see Reference 21 or 22$)$, that $H_{1} \in \mathscr{L}(\hat{\mathscr{X}}, \mathscr{X}), H_{2} \in \mathscr{L}\left(\hat{\mathscr{X}}, \mathbb{R}^{l}\right), R_{1} \in \mathscr{L}(\mathscr{X})$ is (self-adjoint) non-negative definite and that $R_{2}$ is an $m \times m$ (symmetric) positive definite matrix. We define $V_{1}=H_{1} H_{1}^{*}$ and $V_{2}=H_{2} H_{2}^{*}$, where ( $)^{*}$ denotes adjoint, and assume for convenience that $H_{1} H_{2}^{*}=0$ and that $V_{2}$ is positive definite. In addition we make the assumption that either the open-loop semigroup $\{T(t): t \geqslant 0\}$ is Hilbert-Schmidt or the operator $V_{1}$ is trace class. Recall that a linear semigroup $\{S(t): t \geqslant 0\}$ is said to be Hilbert-Schmidt if the operators $S(t)$ are Hilbert-Schmidt for $t>0$. Note also that $H_{1}$ Hilbert-Schmidt is sufficient for $V_{1}$ to be trace class. The compensator is assumed to be of fixed finite order $n_{\mathrm{c}}$ (i.e. $x_{\mathrm{c}}(t) \in \mathbb{R}^{n_{\mathrm{c}}}$ ) and $A_{\mathrm{c}}$, $B_{\mathrm{c}}$ and $C_{\mathrm{c}}$ are matrices of appropriate dimension. For further details and discussion on the problem statement and the above assumptions, see Reference 18. 
We summarize here the primary result from Reference 18 characterizing optimal fixed-finiteorder controllers. For convenience define $\Sigma \triangleq B R_{2}^{-1} B^{*}$ and $\bar{\Sigma} \triangleq C^{*} V_{2}^{-1} C$. Also let $I_{n_{\mathrm{c}}}$ and $I_{\text {., }}$ denote respectively the $n_{\mathrm{c}} \times n_{\mathrm{c}}$ identity matrix and the identity operator on $\mathscr{X}$.

\section{Theorem 1}

Let $n_{\mathrm{c}}$ be given and suppose there exists a controllable and observable $n_{\mathrm{c}}$ th-order dynamic compensator $\left(A_{\mathrm{c}}, B_{\mathrm{c}}, C_{\mathrm{c}}\right)$ which minimizes $J$ given by (5) and for which the closed-loop semigroup generated by

$$
\mathscr{A} \triangleq\left[\begin{array}{cc}
A & B C_{\mathrm{c}} \\
B_{\mathrm{c}} C & A_{\mathrm{c}}
\end{array}\right]
$$

is uniformly exponentially stable. Then there exist non-negative definite operators $Q, P, \hat{Q}, \hat{P}$ on $\mathscr{X}$ such that $A_{\mathrm{c}}, B_{\mathrm{c}}$ and $C_{\mathrm{c}}$ are given by

$$
\begin{gathered}
A_{\mathrm{c}}=\Gamma(A-Q \bar{\Sigma}-\Sigma P) G^{*} \\
B_{\mathrm{c}}=\Gamma Q C^{*} V_{2}^{-1} \\
C_{\mathrm{c}}=-R_{2}^{-1} B^{*} P G^{*}
\end{gathered}
$$

where $\{\mathscr{T}(t): t \geqslant 0\}$ is the closed-loop semigroup on $\mathscr{X} \times \mathbb{R}^{n_{\mathrm{c}}}$ generated by the operator $\mathscr{A}$ given by (6),

$$
\begin{gathered}
\operatorname{rank} \hat{Q}=\operatorname{rank} \hat{P}=\operatorname{rank} \hat{Q} \hat{P}=n_{\mathrm{c}} \\
\hat{Q} \hat{P}=G^{*} M \Gamma, \quad \Gamma G^{*}=I_{n_{\mathrm{c}}}
\end{gathered}
$$

for some $M \in \mathbb{R}^{n_{\mathrm{c}} \times n_{\mathrm{c}}}$,

$$
\begin{aligned}
& 0=A Q+Q A^{*}+V_{1}-Q \bar{\Sigma} Q+\tau_{\perp} Q \bar{\Sigma} Q \tau_{\perp}^{*} \\
& 0=A^{*} P+P A+R_{1}-P \Sigma P+\tau_{\perp}^{*} P \Sigma P{ }_{\perp} \\
& 0=(A-\Sigma P) \hat{Q}+\hat{Q}(A-\Sigma P)^{*}+Q \bar{\Sigma} Q-\tau_{\perp} Q \bar{\Sigma} Q \tau_{\perp}^{*} \\
& 0=(A-Q \bar{\Sigma})^{*} \hat{P}+\hat{P}(A-Q \bar{\Sigma})+P \Sigma P-\tau_{\perp}^{*} P \Sigma P \tau_{\perp}
\end{aligned}
$$

where

$$
\tau \triangleq G^{*} \Gamma, \quad \tau_{\perp} \triangleq I_{2}, \tau
$$

Furthermore, the resulting optimal closed-loop cost is given by

$$
J\left(A_{\mathrm{c}}, B_{\mathrm{c}}, C_{\mathrm{c}}\right)=\operatorname{tr} \int_{0}^{\infty} \mathscr{T}(t) \mathscr{V} \mathscr{T}^{*}(t) \mathscr{R} \mathrm{d} t
$$

where $\{\mathscr{T}(t): t \geqslant 0\}$ is the closed-loop semigroup on $\mathscr{X} \times \mathbb{R}^{n_{\mathrm{c}}}$ generated by the operator $\mathscr{A}$ given by (6)

$$
\begin{gathered}
\mathscr{V} \triangleq\left[\begin{array}{cc}
V_{1} & 0 \\
0 & B_{\mathrm{c}} V_{2} B_{\mathrm{c}}^{\mathrm{T}}
\end{array}\right] \\
\mathscr{R} \triangleq\left[\begin{array}{cc}
R_{1} & 0 \\
0 & C_{\mathrm{c}}^{\mathrm{T}} R_{2} C_{\mathrm{c}}
\end{array}\right]
\end{gathered}
$$

It is shown in Reference 18 that the factorization (11) for the non-negative definite operators $\hat{Q}$ and $\hat{P}$ satisfying rank $\hat{Q} \hat{P}=n_{\mathrm{c}}$ always exists and is unique except for a change of basis in $\mathbb{R}^{n_{\mathrm{c}}}$. Also shown is that $G^{*}: \mathbb{R}^{n_{\mathrm{c}}} \rightarrow \operatorname{Dom}(A)$ so that the expression (7) is well-defined. 
Equations (12)-(15) are, in general, infinite-dimensional operator equations. To actually use them to compute the optimal fixed-order compensator, a finite-dimensional plant approximation is required. For each $N=1,2, \ldots$ let $\mathscr{X}^{N}$ denote a finite-dimensional subspace of $\mathscr{X}$ and let $\mathscr{P}^{N}: \mathscr{X} \rightarrow \mathscr{X}^{N}$ denote the corresponding orthogonal projection of $\mathscr{X}$ onto $\mathscr{X}^{N}$. Let $A^{N} \in \mathscr{L}\left(\mathscr{X}^{N}\right), B^{N} \in \mathscr{L}\left(\mathbb{R}^{m}, \mathscr{X}^{N}\right), C^{N} \in \mathscr{L}\left(\mathscr{X}^{N}, \mathbb{R}^{l}\right), R_{1}^{N} \in \mathscr{L}\left(\mathscr{X}^{N}\right)$ and $V_{1}^{N} \in \mathscr{L}\left(\mathscr{X}^{N}\right)$. We consider the system (7)-(15) with the plant $\Pi$ replaced by the plant $\Pi^{N}$ given by

$$
\Pi^{N} \triangleq\left\{A^{N}, B^{N}, C^{N}, R_{1}^{N}, R_{2}, V_{1}^{N}, V_{2}\right\}
$$

Typically, the operators $B^{N}, C^{N}, R_{1}^{N}$ and $V_{1}^{N}$ are chosen as $B^{N}=\mathscr{P}{ }^{N} B, \quad C^{N}=C \mathscr{P}^{N}$, $R_{1}^{N}=\mathscr{P}^{N} R_{1}$ and $V_{1}^{N}=\mathscr{P}^{N} V_{1}$ with the requirement that $\mathscr{P}^{N}$ converge strongly to the identity $I$, as $N \rightarrow \infty$. The operator $A^{N}$ is chosen so that it and its adjoint satisfy the hypotheses of the Trotter-Kato semigroup approximation theorem (i.e. stability and consistency; see e.g. Reference 23). That is, $A^{N}$ is chosen so that $\lim _{N \rightarrow \infty} T^{N}(t) \mathscr{P}^{N} \phi=T(t) \phi$ and $\lim _{N \rightarrow \infty} T^{N}(t)^{*} \mathscr{P}^{N} \phi=T(t)^{*} \phi$ uniformly in $t$ for $t$ in bounded intervals for each $\phi \in \mathscr{X}$; where $T^{N}(t)=\exp \left(t A^{N}\right), t \geqslant 0$. We shall say more about these choices for $A^{N}, B^{N}, C^{N}, R_{1}^{N}$ and $V_{1}^{N}$ when we make some remarks concerning convergence questions below.

Although with the plant $\Pi^{N}$ equations (12)-(15) are finite-dimensional, they are still operator equations. It is their matrix equivalents which are used in computations. Unless orthonormal bases are chosen for the subspaces $\mathscr{X}^{N}$ (which is typically not the case in practice), some care must be taken to obtain the appropriate matrix system.

For each $N=1,2, \ldots$ let $\left\{\phi_{j}^{N}\right\}_{j=1}^{k^{N}}$ be a basis for $\mathscr{X}^{N}$ and choose the standard bases for all Euclidean spaces. For a linear operator $L$ with domain and range $\mathscr{X}^{N}$ or any Euclidean space, let $[L]$ denote its matrix representation with respect to the bases chosen above. Also, let $\Phi^{N}$ denote the $k^{N}$-square Gram matrix corresponding to the basis $\left\{\phi_{j}^{N}\right\}_{j=1}^{k^{N}}$; that is, $\Phi_{i j}^{n}=\left\{\phi_{i}^{N}, \phi_{j}^{N}\right\rangle, i, j=1,2, \ldots, k^{N}$. Noting that

$$
\begin{gathered}
{\left[\left(A^{N}\right)^{*}\right]=\left(\Phi^{N}\right)^{-1}\left[A^{N}\right]^{\mathrm{T}} \Phi^{N}, \quad\left[\left(B^{N}\right)^{*}\right]=\left[B^{N}\right]^{\mathrm{T}} \Phi^{N}, \quad\left[\left(C^{N}\right)\right]=\left(\Phi^{N}\right)^{-1}\left[C^{N}\right]^{\mathrm{T}}} \\
{\left[\left(\tau_{\perp}^{N}\right)^{*}\right]=\left(\Phi^{N}\right)^{-1}\left[\tau_{\perp}^{N}\right]^{\mathrm{T}} \Phi^{N}, \quad\left[\Sigma^{N}\right]=\left[B^{N}\right] R_{2}^{-1}\left[B^{N}\right]^{\mathrm{T}} \Phi^{N},} \\
{\left[\bar{\Sigma}^{N}\right]=\left(\Phi^{N}\right)^{-1}\left[C^{N}\right]^{\mathrm{T}} V_{2}^{-1}\left[C^{N}\right]}
\end{gathered}
$$

the matrix equivalents of the operator equations (11)-(14) become

$$
\begin{aligned}
0= & {\left[A^{N}\right]\left[Q^{N}\right]+\left[Q^{N}\right]\left(\Phi^{N}\right)^{-1}\left[A^{N}\right]^{\mathrm{T}} \Phi^{N}+\left[V_{1}^{N}\right]-\left[Q^{N}\right]\left[\bar{\Sigma}^{N}\right]\left[Q^{N}\right] } \\
& +\left[\tau_{\perp}^{N}\right]\left[Q^{N}\right]\left[\bar{\Sigma}^{N}\right]\left[Q^{N}\right]-\left(\Phi^{N}\right)^{-1}\left[\tau_{\perp}^{N}\right]^{\mathrm{T}} \Phi^{N} \\
0= & \left(\Phi^{N}\right)^{-1}\left[A^{N}\right]{ }^{\mathrm{T}} \Phi^{N}\left[P^{N}\right]+\left[P^{N}\right]-\left[A^{N}\right]+\left[R_{1}^{N}\right]-\left[P^{N}\right]\left[\Sigma^{N}\right]\left[P^{N}\right] \\
& +\left(\Phi^{N}\right)^{-1}\left[\tau_{\perp}^{N}\right]^{\mathrm{T}} \Phi^{N}\left[P^{N}\right]-\left[\Sigma^{N}\right]\left[P^{N}\right]\left[\tau_{\perp}^{N}\right] \\
0= & \left(\left[A^{N}\right]-\left[\Sigma^{N}\right]\left[P^{N}\right]\right)\left[\hat{Q}^{N}\right]+\left[\hat{Q}^{N}\right]\left(\Phi^{N}\right)^{-1}\left(\left[A^{N}\right]-\left[\Sigma^{N}\right]\left[P^{N}\right]\right)^{\mathrm{T}} \Phi^{N} \\
& +\left[Q^{N}\right]\left[\bar{\Sigma}^{N}\right]\left[Q^{N}\right]-\left[\tau_{\perp}^{N}\right]\left[Q^{N}\right]\left[\bar{\Sigma}^{N}\right]\left[Q^{N}\right]\left(\Phi^{N}\right)^{-1}-\left[\tau_{\perp}^{N}\right]^{\mathrm{T}} \Phi^{N} \\
0= & \left(\Phi^{N}\right)^{-1}\left(\left[A^{N}\right]-\left[Q^{N}\right]\left[\overline{\Sigma^{N}}\right]\right)^{\mathrm{T}} \Phi^{N}\left[\hat{P}^{N}\right]+\left[\hat{P}^{N}\right]\left(\left[A^{N}\right]-\left[Q^{N}\right]\left[\overline{\Sigma^{N}}\right]\right) \\
& +\left[P^{N}\right]\left[\Sigma^{N}\right]\left[P^{N}\right]-\left(\Phi^{N}\right)^{-1}\left[\tau_{\perp}^{N}\right]^{\mathrm{T}} \Phi^{N}\left[P^{N}\right]\left[\Sigma^{N}\right]\left[P^{N}\right]\left[\tau_{\perp}^{N}\right]
\end{aligned}
$$

Therefore, if we define the $k^{N} \times k^{n}$ non-negative definite matrices

$$
\begin{array}{ll}
Q_{0}^{N} \triangleq\left[Q^{N}\right]\left(\Phi^{N}\right)^{-1}, & P_{0}^{N} \triangleq \Phi^{N}\left[P^{N}\right] \\
\hat{Q}_{0}^{N} \triangleq\left[\hat{Q}^{N}\right]\left(\Phi^{N}\right)^{-1}, & \hat{P}_{0}^{N} \triangleq \Phi^{N}\left[\hat{P}^{N}\right] \\
V_{0}^{N} \triangleq\left[V_{1}^{N}\right]\left(\Phi^{N}\right)^{-1}, & R_{0}^{N} \triangleq \Phi^{N}\left[R_{1}^{N}\right] \\
\Sigma_{0}^{N} \triangleq\left[B^{N}\right] R_{2}^{-1}\left[B^{N}\right] & \bar{\Sigma}_{0}^{N} \triangleq\left[C^{N}\right]^{\mathrm{T}} V_{2}^{-1}\left[C^{N}\right]
\end{array}
$$


we can solve the matrix optimal projection equations given in Reference 14 corresponding to the matrix plant model

$$
\Pi_{0}^{N} \triangleq\left\{\left[A^{N}\right],\left[B^{N}\right],\left[C^{N}\right], R_{0}^{N}, R_{2}, V_{0}^{N}, V_{2}\right\}
$$

to obtain the matrices $Q_{0}^{N}, P_{0}^{N}, \hat{Q}_{0}^{N}$ and $\hat{P}_{0}^{N}$. The approximating optimal $n_{\mathrm{c}}$ th-order dynamic compensator $\left\{A_{\mathrm{c}}^{N}, B_{\mathrm{c}}^{N}, C_{\mathrm{c}}^{N}\right\}$ is then given by

$$
\begin{aligned}
& A_{\mathrm{c}}^{N}=\Gamma_{0}^{N}\left(\left[A^{N}\right]-Q_{0}^{N} \bar{\Sigma}_{0}^{N}-\Sigma_{0}^{N} P_{0}^{N}\right)\left(G_{0}^{N}\right)^{\mathrm{T}} \\
& B_{\mathrm{c}}^{N}=\Gamma_{0}^{N} Q_{0}^{N}\left[C^{N}\right]{ }^{\mathrm{T}} V_{2}^{-1} \\
& C_{\mathrm{c}}^{N}=-R_{2}^{-1}\left[B^{N}\right]{ }^{\mathrm{T}} P_{0}^{N}\left(G_{0}^{N}\right)^{\mathrm{T}}
\end{aligned}
$$

where $\Gamma_{0}^{N}, G_{0}^{N} \in \mathbb{R}^{n_{\mathrm{c}}} \times k^{N}$ and $M^{N} \in \mathbb{R}^{n_{\mathrm{c}} \times n_{\mathrm{c}}}$ satisfy

$$
\begin{array}{cc}
\hat{Q}_{0}^{N} \hat{P}_{0}^{N}=G_{0}^{N} M^{N} \Gamma_{0}^{N} & \Gamma_{0}^{N}\left(G_{0}^{N}\right)^{\mathrm{T}}=I_{n_{\mathrm{c}}} \\
{\left[\tau^{N}\right]=\left(G_{0}^{N}\right)^{\mathrm{T}} \Gamma_{0}^{N},} & {\left[\tau_{\perp}^{N}\right]=I_{k}^{N}-\left[\tau^{N}\right]}
\end{array}
$$

When an infinite-dimensional controller will suffice, $C_{\mathrm{c}}=-R_{2}^{-1} B^{*} P \in \mathscr{L}\left(\mathscr{X}, \mathbb{R}^{m}\right)$ and $B_{\mathrm{c}}=Q C^{*} V_{2}^{-1} \in \mathscr{L}\left(\mathbb{R}^{l}, \mathscr{X}\right)$ are the usual infinite-dimensional LQG controller and observer gains. ${ }^{9}$ The operators $P, Q \in \mathscr{L}(\mathscr{X})$ are the non-negative definite solutions to the two decoupled operator algebraic Riccati equations (12) and (13) with $\tau$ and $\tau_{\perp}$ formally set to $I_{\mathscr{S}}$ and 0 respectively. Since $C_{c}$ has range in $\mathbb{R}^{m}$ and $B_{c}$ has domain $\mathbb{R}^{l}$, there exist vectors $c_{\mathrm{c}}=\left(c_{\mathrm{c}}^{1}, \ldots, c_{\mathrm{c}}^{m}\right)^{\mathrm{T}} \in \times_{j=1}^{m} \mathscr{X}$ and $b_{\mathrm{c}}=\left(b_{\mathrm{c}}^{1}, \ldots, b_{\mathrm{c}}^{l}\right)^{\mathrm{T}} \in \times_{j=1}^{l} \mathscr{X}$ such that

$$
\begin{gathered}
{\left[C_{\mathrm{c}} x\right]_{i}=\left\langle c_{\mathrm{c}}^{i}, x\right\rangle, \quad i=1,2, \ldots, m \quad x \in \mathscr{X}} \\
B_{\mathrm{c}} y=b_{\mathrm{c}}^{\mathrm{T}} y=\sum_{i=1}^{l} y_{i} b_{\mathrm{c}}^{i}, \quad y=\left(y_{1}, \ldots, y_{l}\right) \in \mathbb{R}^{l}
\end{gathered}
$$

The vectors $c_{\mathrm{c}}$ and $b_{\mathrm{c}}$ are referred to as the optimal LQG functional controller and observer gains respectively.

With regard to approximation for the full order LQG problem, for each $N=1,2, \ldots$ we take $n_{\mathrm{c}}=k^{N}$. Then it is not difficult to show that

$$
\begin{array}{rlrl}
C_{\mathrm{c}}^{N}\left[P^{N} x\right] & =\left\langle c_{\mathrm{c}}^{N}, x\right\rangle, & & x \in \mathscr{X} \\
B_{\mathrm{c}}^{n} y & =\left(b_{\mathrm{c}}^{N}\right)^{\mathrm{T}} y, & y \in \mathbb{R}^{l}
\end{array}
$$

where $c_{\mathrm{c}}^{N} \in \times_{j=1}^{m} \mathscr{X}^{N}$ and $b_{\mathrm{c}}^{N} \in \times_{j=1}^{l} \mathscr{X}^{N}$ are given by $c_{\mathrm{c}}^{N}=C_{\mathrm{c}}^{N}\left(\Phi^{N}\right)^{-1} \phi^{N}$ and $b_{\mathrm{c}}^{N}=\left(B_{\mathrm{c}}^{N}\right)^{\mathrm{T}} \phi^{N}$ respectively with $\phi^{n}=\left(\phi_{1}^{N}, \ldots, \phi_{k}^{N}\right) \in \times_{j=1}^{k^{N}} \mathscr{X}^{N}$. The vectors $c_{\mathrm{c}}^{N}$ and $b_{\mathrm{c}}^{N}$ are referred to as the approximating optimal LQG functional controller and observer gains respectively. To compute them we need only solve two standard decoupled matrix algebraic Riccati equations for the $k^{N} \times k^{N}$ non-negative definite matrices $Q_{0}^{N}$ and $P_{0}^{N}$.

A rather complete convergence theory for LQG approximation can be found in Reference 9. Essentially, it is shown there that if the approximating subspaces $\mathscr{X}^{N}$ are chosen so that the projections $\mathscr{P}^{N}$ converge strongly to the identity as $N \rightarrow \infty$, the operators $A^{N}, B^{N}, C^{N}, R_{1}^{N}$ and $V_{1}^{N}$ are chosen as was described above and the operators $Q^{N}$ and $P^{N}$ are uniformly bounded in $N$, then $Q^{N}$ and $P^{N}$ converge weakly to $Q$ and $P$ respectively as $N \rightarrow \infty$. This in turn implies that $C_{\mathrm{c}}^{N} \rightarrow C_{\mathrm{c}}$ strongly, $B_{\mathrm{c}}^{N} \rightarrow B_{\mathrm{c}}$ weakly, $c_{\mathrm{c}}^{N} \rightarrow c_{\mathrm{c}}$ and $b_{\mathrm{c}}^{N} \rightarrow b_{\mathrm{c}}$ weakly and the closed-loop semigroup for the approximating optimal LQG compensator converges weakly to the closedloop semigroup for the optimal infinite-dimensional LQG compensator as $N \rightarrow \infty$. If, in addition, the operators $S^{N}(t)=T^{N}(t)+B^{N} C_{\mathrm{c}}^{N}$ and $\hat{S}^{N}(t)=T^{N}(t)-B_{\mathrm{c}}^{N} C^{N}$ are uniformly exponentially stable, uniformly in $N$, then $Q^{N} \rightarrow Q$ and $P^{N} \rightarrow P$ strongly, $C_{\mathrm{c}}^{N} \rightarrow C_{\mathrm{c}}$ and $B_{\mathrm{c}}^{N} \rightarrow B_{\mathrm{c}}$ in norm, $c_{\mathrm{c}}^{N} \rightarrow c_{\mathrm{c}}$ and $b_{\mathrm{c}}^{n} \rightarrow b_{\mathrm{c}}$ strongly and the closed-loop semigroups converge 
strongly as $N \rightarrow \infty$. If $R_{1}^{N}$ and $V_{1}^{N}$ are coercive, uniformly in $N$, then $S^{N}(t)$ and $\hat{S}^{N}(t)$ will be uniformly exponentially stable. If it is also true that $R_{1}$ and $V_{1}$ are trace class and $R_{1}^{N} \mathscr{P}^{N} \rightarrow R_{1}$ and $V_{1}^{N} \mathscr{P}^{N} \rightarrow V_{1}$ in trace norm, then $Q$ and $P$ are trace class and $Q^{N} \mathscr{P}^{N} \rightarrow Q$ and $P^{N} \mathscr{P}^{N} \rightarrow P$ in trace norm as $N \rightarrow \infty$. The development of a complete convergence theory for the approximating fixed-order designs appears to be a much more difficult question. One would expect, however, that any such theory would require at least minimally that the sufficient conditions for convergence of the approximating LQG designs be satisfied.

Returning to the fixed-finite-order case we note that in general the approximating optimal projection equations may not possess a unique solution. However, Richter ${ }^{19}$ shows for the finite-dimensional case that it is possible to obtain an upper bound for the number of stabilizing solutions. He uses topological degree theory to obtain the following result.

\section{Theorem 2}

Consider equations (12)-(15) with the infinite-dimensional plant $\Pi$ replaced by the finitedimensional plant $\Pi^{N}$. Let $n_{\mathrm{u}}$ denote the dimension of the unstable subspace of $A^{N}$ and assume that $n_{\mathrm{c}} \geqslant n_{\mathrm{u}}$. Then in the class of non-negative definite operators $Q^{N}, P^{N}, \hat{Q}^{N}, \hat{P}^{N}$ on $\mathscr{X}^{N}$ satisfying rank $\hat{Q}^{N}=\operatorname{rank} \hat{P}^{N}=\operatorname{rank} \hat{Q}^{N} \hat{P}^{N}=n_{\mathrm{c}}$ there exist at most

$$
\begin{array}{ll}
\left(\begin{array}{cl}
\min \left(k^{N}, m, l\right)-n_{\mathrm{u}} \\
n_{\mathrm{c}}-n_{\mathrm{u}}
\end{array}\right), & n_{\mathrm{c}} \leqslant \min \left(k^{N}, m, l\right) \\
1, & \text { otherwise }
\end{array}
$$

solutions of (12)-(15), each of which is stabilizing. If, in addition, the plant $\left(A^{N}, B^{N}, C^{N}\right)$ is stabilizable by an $n_{\mathrm{c}}$ th-order controller, then there exists at least one stabilizing solution of (10)-(15).

Theorem 2 shows that while there may exist multiple solutions to the finite-dimensional optimal projection equations, in practice this number can be quite small. For example, if $n_{\mathrm{c}} \geqslant n_{\mathrm{u}}$ and the system is either single-input $(m=1)$ or single-output $(l=1)$, then there exists at most one solution to (10)-(15) for the plant $\Pi^{N}$. Moreover, the number of solutions of the approximating optimal projection equations remains bounded in $N$, since for $N$ sufficiently large, $\min \left(k^{N}, m, l\right)=\min (m, l)$. The existence of at least one stabilizing solution of course depends upon whether or not the plant is stabilizable by an $n_{\mathrm{c}}$ th-order controller (for relevant results, see Reference 24). Finally, while it may be possible to stabilize a plant with $n_{\mathrm{c}}<n_{\mathrm{u}}$, this case lies outside the scope of the analysis given in Reference 19.

\section{EXAMPLES AND NUMERICAL RESULTS}

We first consider the one-dimensional, single-input/single-output parabolic (heat/diffusion) control system with Dirichlet boundary conditions given by

$$
\begin{gathered}
\frac{\partial v}{\partial t}(t, \eta)=a \frac{\partial^{2} v}{\partial \eta^{2}}(t, \eta)+b(\eta) u(t)+h_{1}(\eta) w_{1}(t, \eta), \quad 0<\eta<1, \quad t>0 \\
v(t, 0)=0, \quad v(t, 1)=0, \quad t>0 \\
y(t)=\int_{0}^{1} c(\eta) v(t, \eta) \mathrm{d} \eta+h_{2} w_{2}(t), \quad t>0
\end{gathered}
$$


where $a>0$, and $b(\cdot)$ and $c(\cdot)$ are given by

$$
\begin{aligned}
& b(\eta)= \begin{cases}1 /\left(\beta_{2}-\beta_{1}\right), & \beta_{1} \leqslant \eta \leqslant \beta_{2} \\
0, & \text { elsewhere }\end{cases} \\
& c(\eta)= \begin{cases}1 /\left(\gamma_{2}-\gamma_{1}\right), & \gamma_{1} \leqslant \eta \leqslant \gamma_{2} \\
0, & \text { elsewhere }\end{cases}
\end{aligned}
$$

with $0 \leqslant \beta_{1}<\beta_{2} \leqslant 1$ and $0 \leqslant \gamma_{1}<\gamma_{2} \leqslant 1$. In (21) and (23), $h(\cdot) \in L_{\infty}(0,1), w_{1}(t, \cdot) \in L_{2}(0,1)$, almost all $t \in[0, \infty)$ (see Reference 23, p. 314 ), $h_{2}$ is a non-zero constant and $w_{2}(\cdot)$ is unitintensity white noise.

To rewrite (21)-(23) in the form (1), (2), in the usual way we take $\mathscr{X}=L_{2}(0,1)$ endowed with the standard $L_{2}$ inner product, let $x(t)=v(t, \cdot), t \geqslant 0$, define $A$ : $\operatorname{Dom}(A) \subset \mathscr{X} \rightarrow \mathscr{X}$ by $A \phi=a D^{2} \phi$ for $\phi \in \operatorname{Dom} A \triangleq H^{2}(0,1) \cap H_{0}^{1}(0,1)$ and define $B \in \mathscr{L}\left(\mathbb{R}^{1}, \mathscr{X}\right)$ and $C \in \mathscr{L}\left(\mathscr{X}, \mathbb{R}^{1}\right)$ by $B u=b(\cdot) u$ for $u \in \mathbb{R}^{1}$ and $C \phi=\int_{0}^{1} c(\eta) \phi(\eta) \mathrm{d} \eta$ for $\phi \in L_{2}(0,1)$ respectively. Furthermore, let $\hat{\mathscr{X}} \triangleq L_{2}(0,1) \times \mathbb{R}$, set $w(t) \triangleq\left(w_{1}(t, \cdot), w_{2}(t)\right) \in \hat{\mathscr{X}}$ and define $H_{1} \in \mathscr{L}(\hat{\mathscr{X}}, \mathscr{X})$ and $H_{2} \in \mathscr{L}\left(\hat{\mathscr{X}}, \mathbb{R}^{1}\right)$ by $H_{1} z=h_{1}(\cdot) z_{1}$ and $H_{2} z=h_{2} z_{2}$ for $z=\left(z_{1}, z_{2}\right) \in \hat{\mathscr{X}}$ respectively.

It is well known (see e.g. Reference 23 ) that $A$ is closed, densely defined and negative definite. Furthermore, $A$ is the infinitesimal generator of a uniformly exponentially stable, analytic (abstract parabolic) Hilbert-Schmidt semigroup $\{T(t): t \geqslant 0\}$ of bounded, self-adjoint linear operators on $\mathscr{X}$.

We consider a linear spline-based Ritz-Galerkin approximation for the open-loop system. For each $N=2,3, \ldots$ let $\left\{\phi_{j}^{N}\right\}_{j=1}^{N-1}$ be the linear spline ('hat') functions defined on the interval $[0,1]$ with respect to the uniform partition $\{0,1 / N, 2 / N, \ldots, 1\}$, i.e.

$$
\phi_{j}^{N}(\eta)= \begin{cases}N \eta-j+1, & \eta \in[(j-1) / N, j \mid N) \\ j+1-N \eta, & \eta \in[j \mid N,(j+1) / N) \\ 0, & \text { elsewhere on }[0,1]\end{cases}
$$

$j=1,2, \ldots, N-1$. Set $\mathscr{X}^{N}=\operatorname{span}\left\{\phi_{j}^{N}\right\}_{j=1}^{N-1}$ and note that $k^{N}=\operatorname{dim} \mathscr{X}^{N}=N-1$ and $\mathscr{X}^{N} \subset H_{0}^{1}(0,1)$ for all $N$. If $\mathscr{P}^{N}: \mathscr{X} \rightarrow \mathscr{X}^{N}$ denotes the orthogonal projection of $\mathscr{X}$ onto $\mathscr{X}^{N}$, then standard convergence estimates for interpolatory splines ${ }^{25}$ can be used to show that $\lim _{N \rightarrow \infty} \mathscr{P}^{N} \phi=\phi$ in $L_{2}(0,1)$ for $\phi \in L_{2}(0,1)$.

There are two equivalent ways to obtain an operator representation for the usual Ritz-Galerkin approximation to $A$. First, $A$ can be extended to a bounded linear operator from $H_{0}^{1}(0,1)$ onto its dual, $H^{-1}(0,1)$, via

$$
(A \phi)(\psi)=-a\langle D \phi, D \psi\rangle, \quad \phi, \psi \in H_{0}^{1}(0,1)
$$

Since $\mathscr{X}^{N} \subset H_{0}^{1}(0,1)$ for all $N=2,3, \ldots$, we define $A^{N} \in \mathscr{L}\left(\mathscr{X}^{N}\right)$ by $A^{N} \phi^{N}=A \phi^{N}, \phi^{N} \in \mathscr{X}^{N}$, with $A \phi^{N} \in H^{-1}(0,1)$ considered to be linear functional on $\mathscr{X}^{N}$. From the Riesz representation theorem we obtain $A^{N} \phi^{N}=\psi^{N}$ where $\psi^{N}$ is that element in $\mathscr{X}^{N}$ which satisfies $\left(A^{N} \phi^{N}\right)\left(\chi^{N}\right)$ $=-a\left\langle D \phi^{N}, D \chi^{N}\right\rangle=\left\langle\psi^{N}, \chi^{N}\right\rangle$.

Alternatively and equivalently, by using the fact that $A$ is self-adjoint, we can define $A^{N}$ as follows. Let $\mathscr{P}_{1}^{N}: H_{0}^{1}(0,1) \rightarrow \mathscr{X}^{N}$ denote the orthogonal projection of the Hilbert space $H_{0}^{1}(0,1)$ onto $\mathscr{X}^{N}$. Using the definition (24) it is not difficult to show that $-A \in \mathscr{L}\left(H_{0}^{1}(0,1), H^{-1}(0,1)\right)$ is coercive and therefore that $A^{-1}: H^{-1}(0,1) \rightarrow H_{\vartheta}^{1}(0,1)$ exists and is bounded. We then define $A^{N} \in \mathscr{L}\left(\mathscr{X}^{N}\right)$ to be the inverse of the operator given by $\left(A^{N}\right)^{-1}=\left.\mathscr{P}_{1}^{N} A^{-1}\right|_{\mathscr{X}^{N}}$.

Using either definition it is easily argued that $A^{N}$ is well-defined, self-adjoint and is the infinitesimal generator of a uniformly exponentially stable (uniformly in $N$ ) semigroup, 
$T^{N}(t)=\exp \left(t A^{N}\right), t \geqslant 0$, of bounded linear operators on $\mathscr{X}^{N}$. Also, using the approximation properties of splines it is not difficult to show that $\lim _{N \rightarrow \infty}\left(A^{N}\right)^{-1} \mathscr{P}^{N} \phi=A^{-1} \phi, \phi \in \mathscr{X}$. Consequently, the hypotheses of the Trotter-Kato theorem ${ }^{23}$ are satisfied and we have $\lim _{N \rightarrow \infty} T^{N}(t) \mathscr{P}^{N} \phi=T(t) \phi$ and $\lim _{N \rightarrow \infty} T^{N}(t)^{*} \mathscr{P}^{N} \phi=T(t)^{*} \phi, \phi \in \mathscr{X}$, uniformly in $t$ for $t$ in bounded intervals. A detailed discussion of the results just outlined can be found in Reference 8 .

We define $B^{N}=\mathscr{P}^{N} B$ and $C^{N}=C \mathscr{P}^{N}$, from which it immediately follows that $\lim _{N \rightarrow \infty} B^{N}$ $=B$ and $\lim _{N \rightarrow \infty} C^{N}=C$ in norm and similarly for their adjoints. For the example we shall consider here, we have chosen $R_{1}=r_{1} I_{\mathscr{X}}$. and $R_{2}=r_{2} I_{m}$, with $r_{1}, r_{2}>0$. Setting $h_{1}(\eta)=v_{1}^{1 / 2}$, $0<\eta<1$, and $h_{2}=v_{2}^{1 / 2}$, with $v_{1}, v_{2}>0$, we obtain $V_{1}=v_{1} I_{2}$ and $V_{2}=v_{2}$. We then take $R_{1}^{N}=\mathscr{P}^{N} R_{1}$ and $V_{1}^{N}=\mathscr{P}^{N} V_{1}$. For the LQG problem the open-loop uniform exponential stability of both the infinite-dimensional system and the approximating systems is sufficient to conclude the strong convergence of the approximating Riccati operators to the unique solutions of the infinite-dimensional Riccati equations, the uniform norm convergence of the approximating controller and observer gains and the strong convergence of the functional gains as $N \rightarrow \infty .^{2,7,9}$

Since the basis elements $\left\{\phi_{j}^{N}\right\}_{j=1}^{N-1}$ are piecewise linear with respect to the uniform mesh $\{0,1 / N, 2 / N, \ldots, 1\}$ on $[0,1]$, the equivalent matrix representations for the operators defined above can be computed directly and in closed form. The Gram matrix $\Phi_{i j}^{N}=\left\langle\phi_{i}^{N}, \phi_{j}^{N}\right\rangle$, $i, j=1,2, \ldots, N-1$, is given by $\Phi^{N}=(1 / N)$ Tridiag $\left\{\frac{1}{6}, \frac{2}{3}, \frac{1}{6}\right\}$, and if we define the generalized stiffness matrix $\Psi^{N}$ by $\Psi_{i j}^{N}=-a\left\langle D \phi_{i}^{N}, D \phi_{j}^{N}\right\rangle, i, j=1,2, \ldots, N-1$, then $\Psi^{N}=a N$ Tridiag $\{1,-2,1\}$. It follows that $\left[A^{N}\right]=\left(\Phi^{N}\right)^{-1} \Psi^{N},\left[B^{N}\right]=\left(\Phi^{N}\right)^{-1} b^{N}$ and $\left[C^{N}\right]=c^{N}$, with $b_{i}^{N}=\left\langle b, \phi_{i}^{N}\right\rangle=$ $\left[1 /\left(\beta_{2}-\beta_{1}\right)\right] \int_{\beta_{1}^{2}}^{\beta_{2}} \phi_{i}^{N}(\eta) \mathrm{d} \eta$ and $c_{i}^{N}=\left\langle c, \phi_{i}^{N}\right\rangle=\left[1 /\left(\gamma_{2}-\gamma_{1}\right)\right] \int_{\gamma_{1}}^{\gamma_{2}} \phi_{i}^{N}(\eta) \mathrm{d} \eta, i=1,2, \ldots, N-1$, and that $R_{0}^{N}=r_{1} \Phi^{N}$ and $V_{0}^{N}=v_{1}\left(\Phi^{N}\right)^{-1}$.

For our numerical study we set $a=1, \beta_{1}=0 \cdot 75-0 \cdot 03 \sqrt{2}, \quad \beta_{2}=0 \cdot 75+0 \cdot 04 \sqrt{2}$, $\gamma_{1}=0 \cdot 25-0 \cdot 04 \sqrt{ } 2, \gamma_{2}=0 \cdot 25+0 \cdot 03 \sqrt{ } 2, r_{1}=v_{1}=1, r_{2}=v_{2}=10^{-4}$ and $h_{1}(\eta) \equiv 1$, and used our technique to compute approximating optimal LQG (i.e. $n_{\mathrm{c}}=N-1$ ) and first-order (i.e. $n_{\mathrm{c}}=1$ ) compensators for various values of $N$. The open-loop stability of system (21)-(23) and the approximating systems implies that the finite-dimensional approximating optimal projection equations have a solution. Theorem 2, on the other hand, with $n_{\mathrm{u}}=0$ and $n_{\mathrm{c}}=1$ or $n_{\mathrm{c}}=N-1$, implies that they have at most one solution. Consequently, the system of equations (12)-(15) with the plants $\Pi^{N}$ admits a unique solution.

The optimal projection equations (12)-(15) were solved by using the homotopic continuation algorithm described in Reference 19. There it is shown that the operation count for the algorithm is proportional to $p\left(2 n^{3}+(m+l) n^{2}+(m+l)^{3} n_{\mathrm{c}}^{3}\right)$, where $p$ is the number of integration steps and $n$ is the dimension of the finite-dimensional plant. This count is competitive with the operation count for the Hamiltonian solution of the standard Riccati equations, which is $O\left(16 n^{3}\right)$ for LQG. Also, note that the computational burden for the solution of the optimal projection equations decreases with $n_{\mathrm{c}}$.

Since $m=l=1$ in the LQG case, the optimal functional observer and feedback control gains $b_{\mathrm{c}}$ and $c_{\mathrm{c}}$ and the approximating gains $b_{\mathrm{c}}^{N}$ and $c_{\mathrm{c}}^{N}$ are all simply $L_{2}$ functions with $b_{\mathrm{c}}^{N}$ and $c_{\mathrm{c}}^{N}$ elements in $\mathscr{X}^{N}$. We plot the functions $b_{\mathrm{c}}^{N}$ and $c_{\mathrm{c}}^{N}$ we obtained for various values of $N$ in Figures 1 and 2 respectively. The apparent symmetry of the plots given in Figures 1 and 2 is a result of the nearly symmetric placement of the sensor and actuator (i.e. the choice of $\beta_{1}, \beta_{2}, \gamma_{1}$ and $\gamma_{2}$ ) in this particular example. That convergence is indeed achieved can immediately be observed in the figures. In the fixed-order case with $n_{\mathrm{c}}=1$, the compensator gains $A_{\mathrm{c}}, B_{\mathrm{c}}$ and $C_{\mathrm{c}}$ are all scalars. Also, for a first-order controller there are only two independent parameters, $A_{\mathrm{c}}$ and $B_{\mathrm{c}} C_{\mathrm{c}}$. In Table 1 we give the values we obtained for $A_{\mathrm{c}}^{N}$ and 


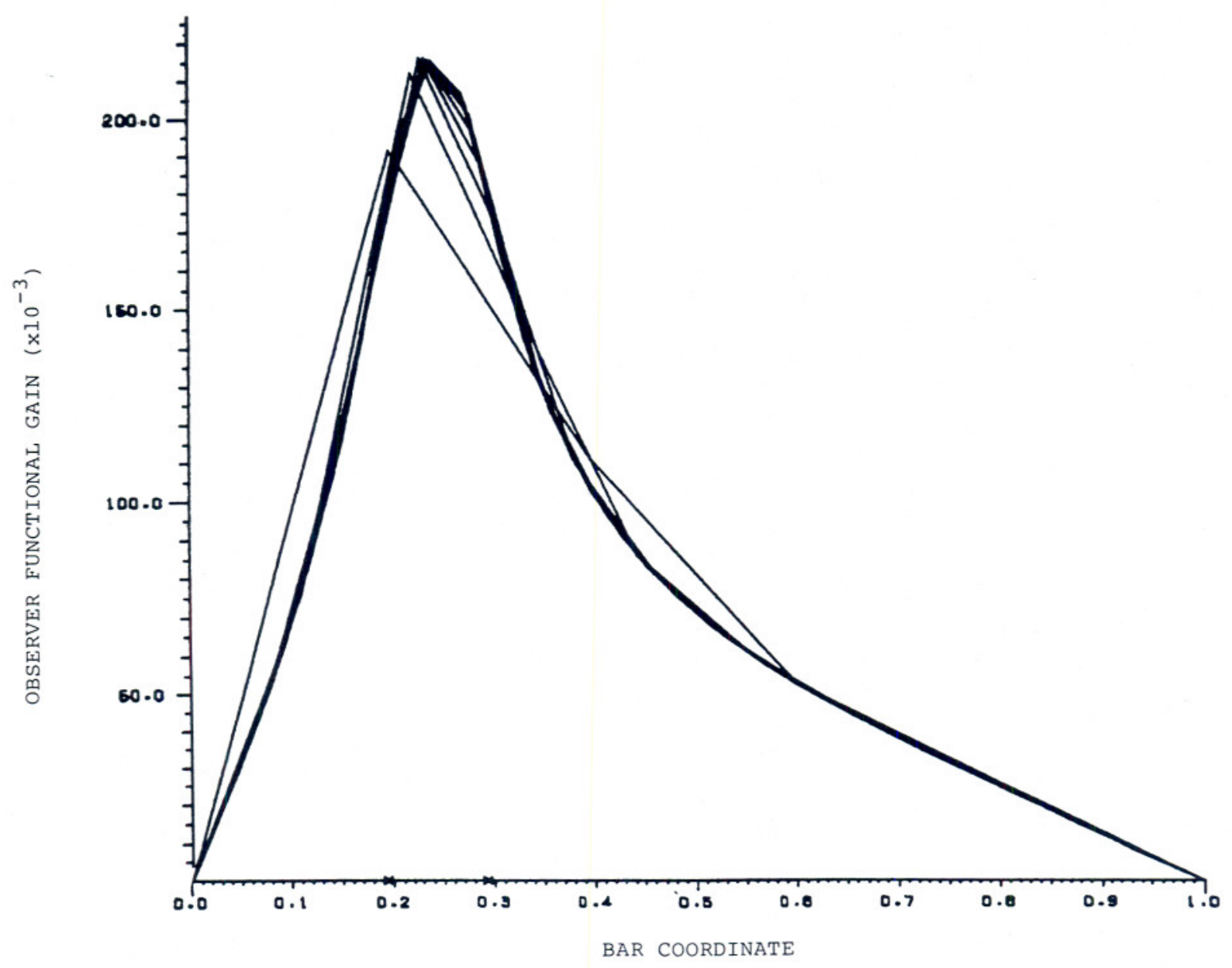

Figure 1. Parabolic system approximating optimal LQG functional observer gains; $N=4,8,12,16,20,24,28,32$

$B_{\mathrm{c}}^{N} C_{\mathrm{c}}^{N}$ for various values of $N$. Once again it is clear that the gains are converging as $N$ increases. In addition, in Table 1 we provide the closed-loop costs $J_{\mathrm{LQG}}^{N}$ and $J_{\mathrm{FO}}^{N}$ computed via formula (16) for the LQG and first-order controllers. These closed-loop costs are evaluated using a 64th-order modal approximation to the infinite-dimensional system. For all values of $N$ the performance of the fixed-order compensator was within $2 \%$ of the corresponding LQG controller. Thus, for example, the replacement of an approximating optimal LQG controller of any desired order by an approximating optimal first-order controller can yield considerable implementation simplification with only minor performance degradation. Note that for the example we consider here it is possible to compute the open-loop cost for the infinitedimensional system in closed form. We have

$$
\begin{aligned}
J_{\mathrm{OL}} & =\operatorname{tr} \int_{0}^{\infty} V_{1} T^{*}(t) R_{1} T(t) \mathrm{d} t=v_{1} r_{1} \operatorname{tr} \int_{0}^{\infty} T(t)^{2} \mathrm{~d} t \\
& =v_{1} r_{1} \sum_{n=1}^{\infty} \int_{0}^{\infty} \exp \left(-2 n^{2} \pi^{2} a t\right) \mathrm{d} t=\frac{v_{1} r_{1}}{2 \pi^{2} a} \sum_{n=1}^{\infty} \frac{1}{n^{2}} \\
& =\frac{v_{1} r_{1}}{12 a}=\frac{1}{12} \approx 0.08333
\end{aligned}
$$




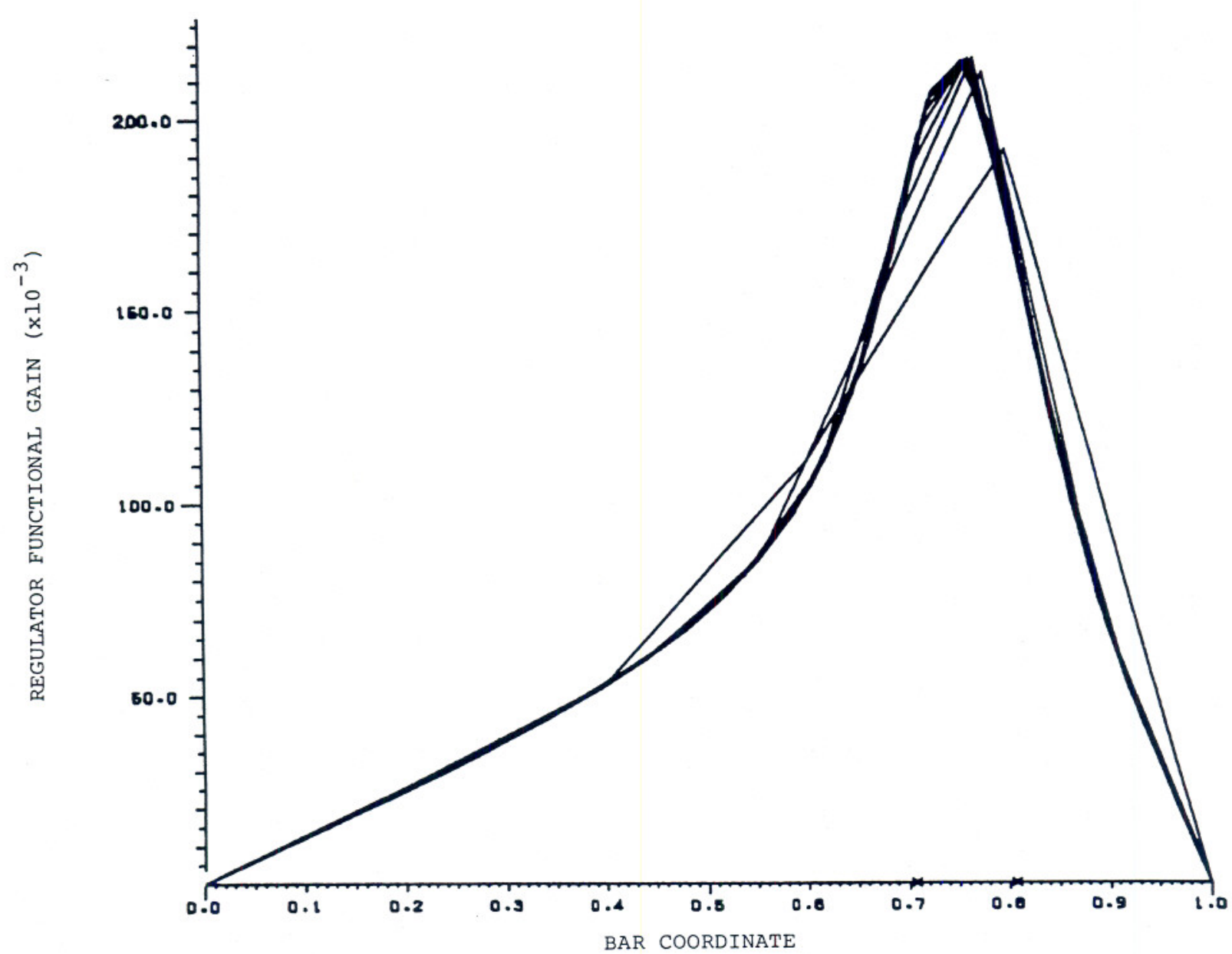

Figure 2. Parabolic system approximating optimal LQG functional control gains; $N=4,8,12,16,20,24,28,32$

Table I. Parabolic system approximating optimal first-order compensator gains

\begin{tabular}{rcccc}
\hline$N$ & \multicolumn{1}{c}{$A_{\mathrm{c}}^{N}$} & $B_{\mathrm{c}}^{N} C_{\mathrm{c}}^{N}$ & $J_{\text {LQG }}^{N}$ & $J_{\text {FO }}^{N}$ \\
\hline 4 & $-687 \cdot 6$ & 5470 & $0 \cdot 06999$ & $0 \cdot 07014$ \\
8 & $-720 \cdot 9$ & 5231 & $0 \cdot 06870$ & $0 \cdot 06993$ \\
12 & $-730 \cdot 9$ & 5182 & $0 \cdot 06872$ & $0 \cdot 06991$ \\
16 & $-734 \cdot 3$ & 5145 & $0 \cdot 06874$ & $0 \cdot 06990$ \\
20 & $-738 \cdot 0$ & 5127 & $0 \cdot 06875$ & $0 \cdot 06990$ \\
24 & $-737 \cdot 6$ & 5108 & $0 \cdot 06876$ & $0 \cdot 06990$ \\
28 & $-739 \cdot 8$ & 5109 & $0 \cdot 06876$ & $0 \cdot 06990$ \\
32 & $-738 \cdot 7$ & 5099 & $0 \cdot 06877$ & $0 \cdot 06990$ \\
\hline
\end{tabular}

Finally, for comparison purposes, we tried applying balancing techniques to the LQG controllers to reduce their order. However, with $n_{\mathrm{c}}=1$, such controllers were found to be destabilizing. On the basis of the results in Reference 13, this was not unexpected. Furthermore, a first-order controller based upon a truncated model consisting of the first mode only was found to yield an unstable closed-loop system for the 64th-order truth model. 
As a second example we consider the one-dimensional, single-input/single-output hereditary control system given by

$$
\begin{gathered}
\dot{v}(t)=a_{0} v(t)+a_{1} v(t-\rho)+b_{0} u(t)+h_{1} w(t), \quad t>0 \\
y(t)=c_{0} v(t)+h_{2} w(t), \quad t>0
\end{gathered}
$$

where $a_{0}, a_{1}, b_{0}, c_{0}, h_{1}, h_{2}, \rho \in \mathbb{R}^{1}$ with $\rho>0, h_{2} \neq 0$, and $w$ is a unit-intensity white noise process. To rewrite (25), (26) in the form (1), (2), we take $\mathscr{X}=\mathbb{R}^{2} \times L_{2}(-\rho, 0)$ endowed with the usual product space inner product, $\langle(\eta, \phi),(\xi, \psi)\rangle=\eta \xi+\int_{-\rho}^{0} \phi \psi$, and let $x(t)=\left(v(t), v_{t}\right)$, $t \geqslant 0$, where for $t \geqslant 0, v_{t} \in L_{2}(-\rho, 0)$ is given by $v_{t}(\theta)=v(t+\theta),-\rho \leqslant \theta \leqslant 0$. Define $A$ : $\operatorname{Dom}(A) \subset \mathscr{X} \rightarrow \mathscr{X} \quad$ by $A \hat{\phi}=\left(a_{0} \phi(0)+a_{1} \phi(-\rho), D \phi\right) \quad$ for $\quad \hat{\phi}=(\phi(0), \phi) \in \operatorname{Dom}(A)$ $\triangleq\left\{(\xi, \psi) \in \mathscr{X}: \psi \in H^{1}(-\rho, 0), \psi(0)=\xi\right\}$ and let $B \in \mathscr{L}\left(\mathbb{R}^{1}, \mathscr{X}\right)$ and $C \in \mathscr{L}\left(\mathscr{X}, \mathbb{R}^{1}\right)$ be given by $B u=\left(b_{0} u, 0\right)$ and $C(\eta, \phi)=c_{0} \eta$ respectively. Let $\hat{\mathscr{X}}=\mathbb{R}^{1}$ and define $H_{1} \in \mathscr{L}(\hat{\mathscr{X}}, \mathscr{X})$ and $H_{2} \in \mathscr{L}\left(\hat{\mathscr{X}}, \mathbb{R}^{1}\right)$ by $H_{1} z=\left(h_{1} z, 0\right)$ and $H_{2} z=h_{2} z$ respectively for $z \in \mathbb{R}^{1}$.

The operator $A$ is densely defined and is the infinitesimal generator of a $C_{0}$ semigroup $\{T(t)$ : $t \geqslant 0\}$ of bounded linear operators on $\mathscr{X}$ with $T(t)(\eta, \phi)=\left(v(t ; \eta, \phi), v_{t}(\eta, \phi)\right), t \geqslant 0$, where $v(\cdot ; \eta, \phi)$ is the unique solution to (25) with $b_{0}=h_{1}=0$ and initial conditions $v(0)=\eta, v_{0}=\phi$. We take $R_{1} \in \mathscr{L}(\mathscr{X})$ and $R_{2} \in \mathscr{L}\left(\mathbb{R}^{1}\right)$ to be $R_{1}(\eta, \phi)=\left(r_{1} \eta, 0\right)$ and $R_{2} u=r_{2} u$ respectively with $r_{1}, r_{2}>0$. The definitions of $H_{1}$ and $H_{2}$ given above imply that $V_{1} \in \mathscr{L}(\mathscr{X})$ and $V_{2} \in \mathscr{L}\left(\mathbb{R}^{1}\right)$ are given by $V_{1}(\eta, \phi)=\left(h_{1}^{2} \eta, 0\right)$ and $V_{2} z=h_{2}^{2} z$ respectively for $(\eta, \phi) \in \mathscr{X}$ and $z \in \mathbb{R}^{1}$. Although in this example the open-loop semigroup $\{T(t): t \geqslant 0\}$ is not compact, the operator $H_{1}$ is of finite rank and therefore Hilbert-Schmidt. The operator $V_{1}$ is thus trace class.

We employ an appropriate scheme recently proposed by Ito and Kappel. ${ }^{26}$ We briefly outline it here; a more detailed discussion can be found in Reference 26. For each $N=1,2, \ldots$ let $\chi_{j}^{N} \in L_{2}(-\rho, 0)$ denote the characteristic function for the interval $[-j \rho / N,-(j-1) \rho / N)$, $j=1,2, \ldots, N$, and let $\mathscr{X}^{N}$ be the $(N+1)$-dimensional subspace of $\mathscr{X}$ defined by

$$
\mathscr{X}^{N}=\operatorname{span}\left\{(1,0),\left(0, \chi_{1}^{N}\right), \ldots,\left(0, \chi_{N}^{N}\right)\right\}
$$

Let $\mathscr{P}^{N}: \mathscr{X} \rightarrow \mathscr{X}^{N}$ denote the orthogonal projection of $\mathscr{X}$ onto $\mathscr{X}^{N}$. Let $\left\{\phi_{j}^{N}\right\}_{j=0}^{N}$ denote the linear $B$-spline functions defined on the interval $[-\rho, 0]$ with respect to the uniform mesh $[-\rho, \ldots,-\rho / N, 0\}$ and set $\mathscr{X}_{1}^{N}=\operatorname{span}\left\{\left(\phi_{j}^{N}(0), \phi_{j}^{N}\right)\right\}_{j=0}^{N}$. Then $\mathscr{X}_{1}^{N}$ is an $(N+1)$-dimensional subspace of $\operatorname{Dom}(A)$ and is not difficult to demonstrate that the restriction of $\mathscr{P}^{N}$ to $\mathscr{X}_{1}^{N}$ is a bijection into $\mathscr{X}^{N}$. Using the fact that $A$ restricted to $\mathscr{X}_{1}^{N}$ has range in $\mathscr{X}^{N}$, we define $A^{N} \in \mathscr{L}\left(\mathscr{X}^{N}\right)$ by $A^{N}=A\left(\mathscr{P}^{N}\right)^{-1}$ and set $T^{N}(t)=\exp \left(A^{N} t\right), t \geqslant 0$. Noting that $R(B) \subset \mathscr{P}^{N}$, we take $B^{N} \in \mathscr{L}\left(\mathbb{R}^{1}, \mathscr{X}^{N}\right)$ to be given by $B^{N}=B$. Similarly, we take $R_{1}^{N}=R_{1}$ and $V_{1}^{N}=V_{1}$. We set $C^{N}=C$.

It is shown in Reference 26 that $\mathscr{P}^{N}(\eta, \phi) \rightarrow(\eta, \phi), T^{N}(t) \mathscr{P}^{N}(\eta, \phi) \rightarrow T(t)(\eta, \phi)$ and $T^{N}(t)^{*} \mathscr{P}^{N}(\eta, \phi) \rightarrow T(t)^{*}(\eta, \phi)$ for $(\eta, \phi) \in \mathscr{X}$ as $N \rightarrow \infty$, uniformly in $t$, for $t$ in bounded subsets of $[0, \infty)$. It then follows that $\lim _{N \rightarrow \infty} B^{N}=B$ and $\lim _{N \rightarrow \infty} C^{N} \mathscr{P}^{N}=C$ in norm.

For the LQG (full-order) problem the optimal functional observer and feedback control gains $b_{\mathrm{c}}$ and $c_{\mathrm{c}}$ are of the form $b_{\mathrm{c}}=\left(\beta_{0}, \beta_{1}\right)$ and $c_{\mathrm{c}}=\left(\gamma_{0}, \gamma_{1}\right)$ respectively with $\beta_{0}, \gamma_{0} \in \mathbb{R}^{1}$ and $\beta_{1}, \gamma_{1} \in L_{2}(-\rho, 0)$. The approximating gains are of the form $b_{\mathrm{c}}^{N}=\left(\beta_{0}^{N}, \beta_{1}^{N}\right)$ and $c_{\mathrm{c}}^{N}=\left(\gamma_{0}^{N}, \gamma_{1}^{N}\right)$ with $\beta_{0}^{N}, \gamma_{0}^{N} \in \mathbb{R}^{1}$ and $\beta_{1}^{N}, \gamma_{1}^{N} \in \operatorname{span}\left\{\chi_{j}^{N}\right\}_{j=1}^{N}$. Since we are treating a one-dimensional example, if $b_{0} \neq 0$, the theory in Reference 26 implies that $\beta_{0}^{N} \rightarrow \beta_{0}$ and $\gamma_{0}^{N} \rightarrow \gamma_{0}$ in $\mathbb{R}^{1}$ and that $\beta_{1}^{N} \rightarrow \beta_{1}$ and $\gamma_{1}^{N} \rightarrow \gamma_{1}$ in $L_{2}(-\rho, 0)$ as $N \rightarrow \infty$.

Once again, as in the first example, matrix representations for the operators $A^{N}, B^{N}, C^{N}, R_{1}^{N}$ and $V_{1}^{N}$ are not difficult to compute in closed form. Indeed, the $(N+1) \times(N+1)$ matrix 
representation for the bijection $\mathscr{P}^{N}: \mathscr{X}_{1}^{N} \rightarrow \mathscr{X}_{1}$ is given by

$$
\left[\mathscr{P}^{N}\right]=\left[\begin{array}{ccccccccc}
1 & 0 & & & & & 0 & \\
\frac{1}{2} & \frac{1}{2} & 0 & & & & & \\
0 & \frac{1}{2} & \frac{1}{2} & 0 & & & & & \\
& & & & \ddots & & & & \\
& & & & 0 & \frac{1}{2} & \frac{1}{2} & 0 \\
& 0 & & & & 0 & \frac{1}{2} & \frac{1}{2}
\end{array}\right]
$$

Then $\left[A^{N}\right]=\left[K^{N}\right]\left[\mathscr{P}^{N}\right]^{-1}$ where

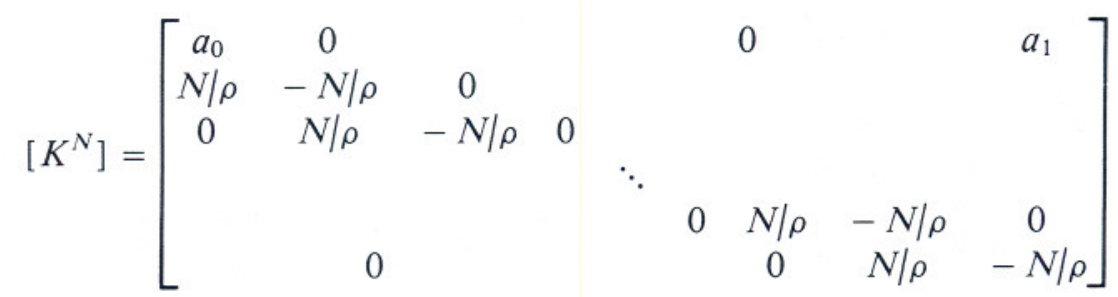

We have the $(N+1) \times 1$ matrix $\left[B^{N}\right]=\left[\begin{array}{lll}b_{0} & 0 \ldots 0\end{array}\right]^{\mathrm{T}}$ and the $1 \times(N+1)$ matrix $\left[C^{N}\right]=$

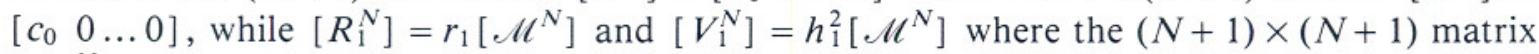
$\left[\mathscr{M}^{N}\right]$ is given by

$$
\left[\mathscr{U}^{N}\right]=\left[\begin{array}{llll}
1 & 0 & & 0 \\
0 & & & 0 \\
& & \ddots & \\
0 & & & 0
\end{array}\right]
$$

We $\quad$ set $\quad a_{0}=a_{1}=b_{0}=c_{0}=r_{1}=h_{1}=\rho=1, \quad r_{2}=0 \cdot 1 \quad$ and $\quad h_{2}=\sqrt{(}(0 \cdot 1)$ and computed approximating optimal LQG (i.e. $n_{\mathrm{c}}=N+1$ ) and first-order (i.e. $n_{\mathrm{c}}=1$ ) compensators for $N=8,16,24$ and 32. The optimal LQG observer gains are given in Table 3 and Figure 3; the control gains are given in Table 4 and Figure 4 . The symmetry in the observer and control gains

Table II. Hereditary system open-loop poles

$$
\begin{aligned}
& 1 \cdot 278465 \\
& -1 \cdot 588317 \pm 4 \cdot 155305 \mathrm{i} \\
& -2 \cdot 417631 \pm 10 \cdot 68603 \mathrm{i} \\
& -2 \cdot 861502 \pm 17 \cdot 05611 \mathrm{i} \\
& -3 \cdot 167754 \pm 23 \cdot 38558 \mathrm{i} \\
& -3 \cdot 401945 \pm 29 \cdot 69798 \mathrm{i} \\
& -3 \cdot 591627 \pm 36 \cdot 00146 \mathrm{i} \\
& -3 \cdot 751047 \pm 42 \cdot 29965 \mathrm{i} \\
& -3 \cdot 888543 \pm 48 \cdot 59442 \mathrm{i} \\
& -4 \cdot 009422 \pm 54 \cdot 88686 \mathrm{i} \\
& -4 \cdot 117267 \pm 61 \cdot 17761 \mathrm{i} \\
& -4 \cdot 214618 \pm 67 \cdot 46710 \mathrm{i}
\end{aligned}
$$

Table III. Hereditary system approximating optimal LQG scalar observer gains

\begin{tabular}{ccccc}
\hline$N$ & 8 & 16 & 24 & 32 \\
\hline$\beta_{0}^{N}$ & $4 \cdot 4213$ & $4 \cdot 4229$ & $4 \cdot 4233$ & $4 \cdot 4234$ \\
\hline
\end{tabular}




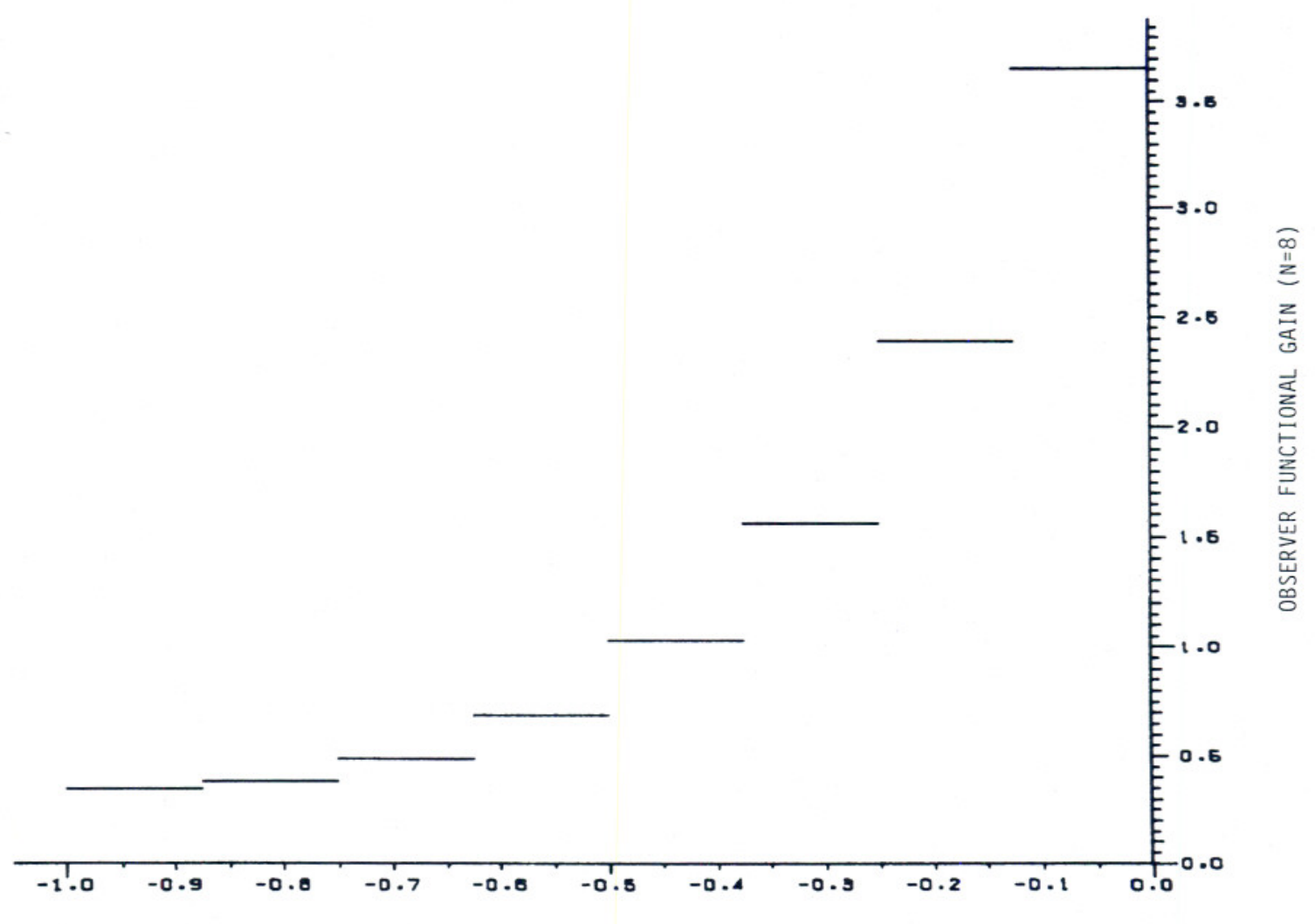

DELAY COORDINATE

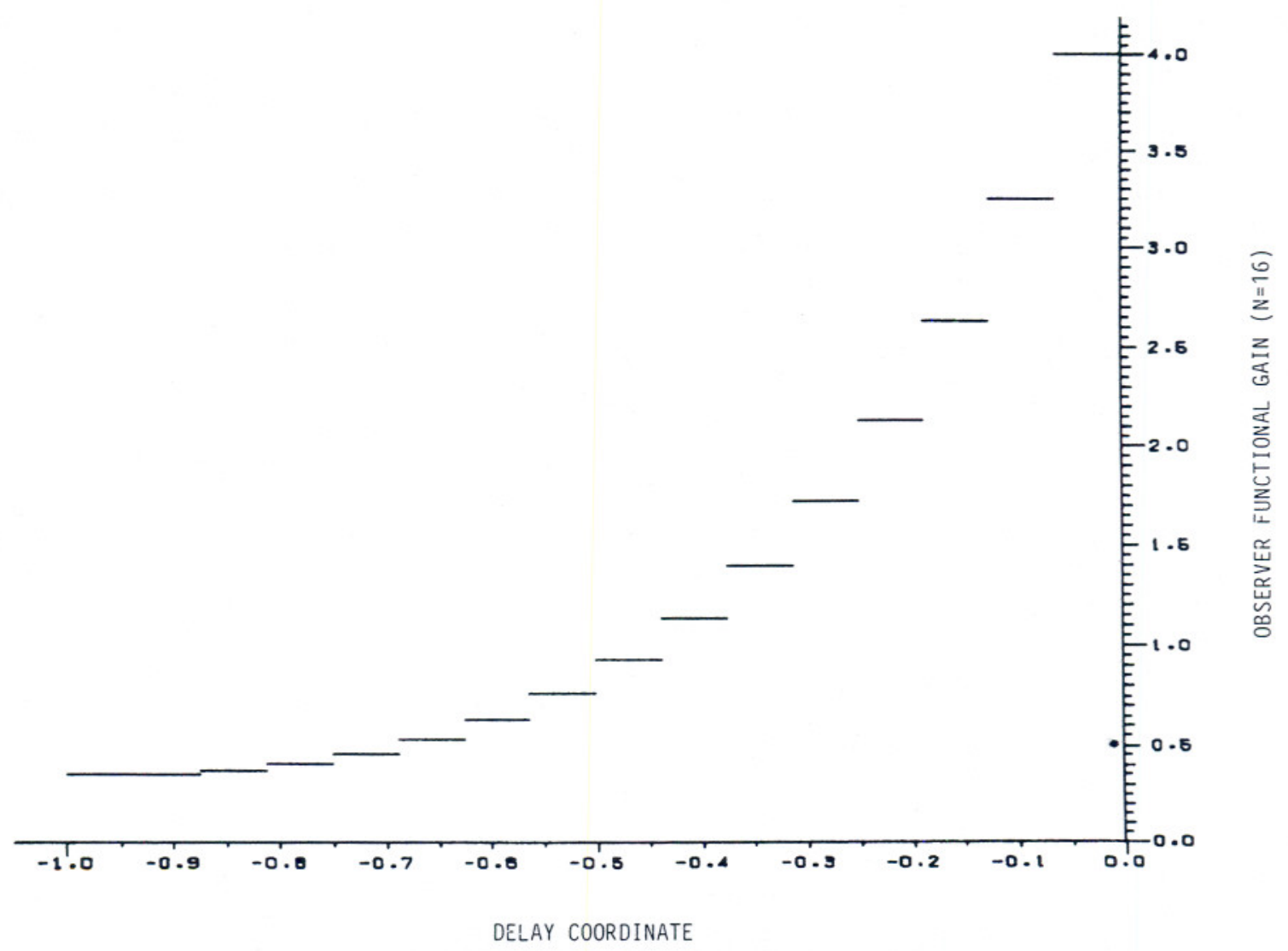



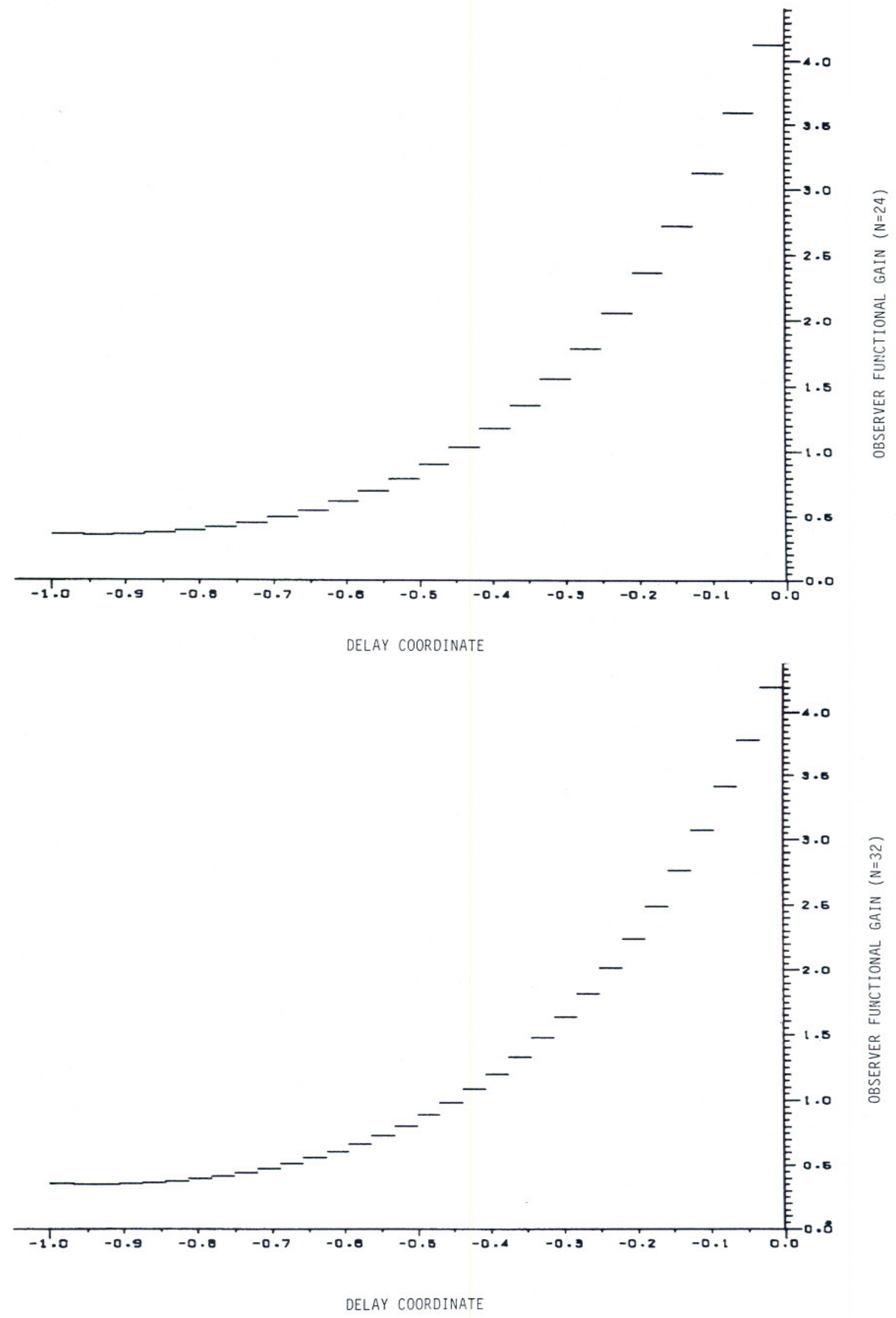

Figure 3. Hereditary system approximating optimal LQG functional observer gains; $N=8,16,24,32$ 
DELAY COORDINATE

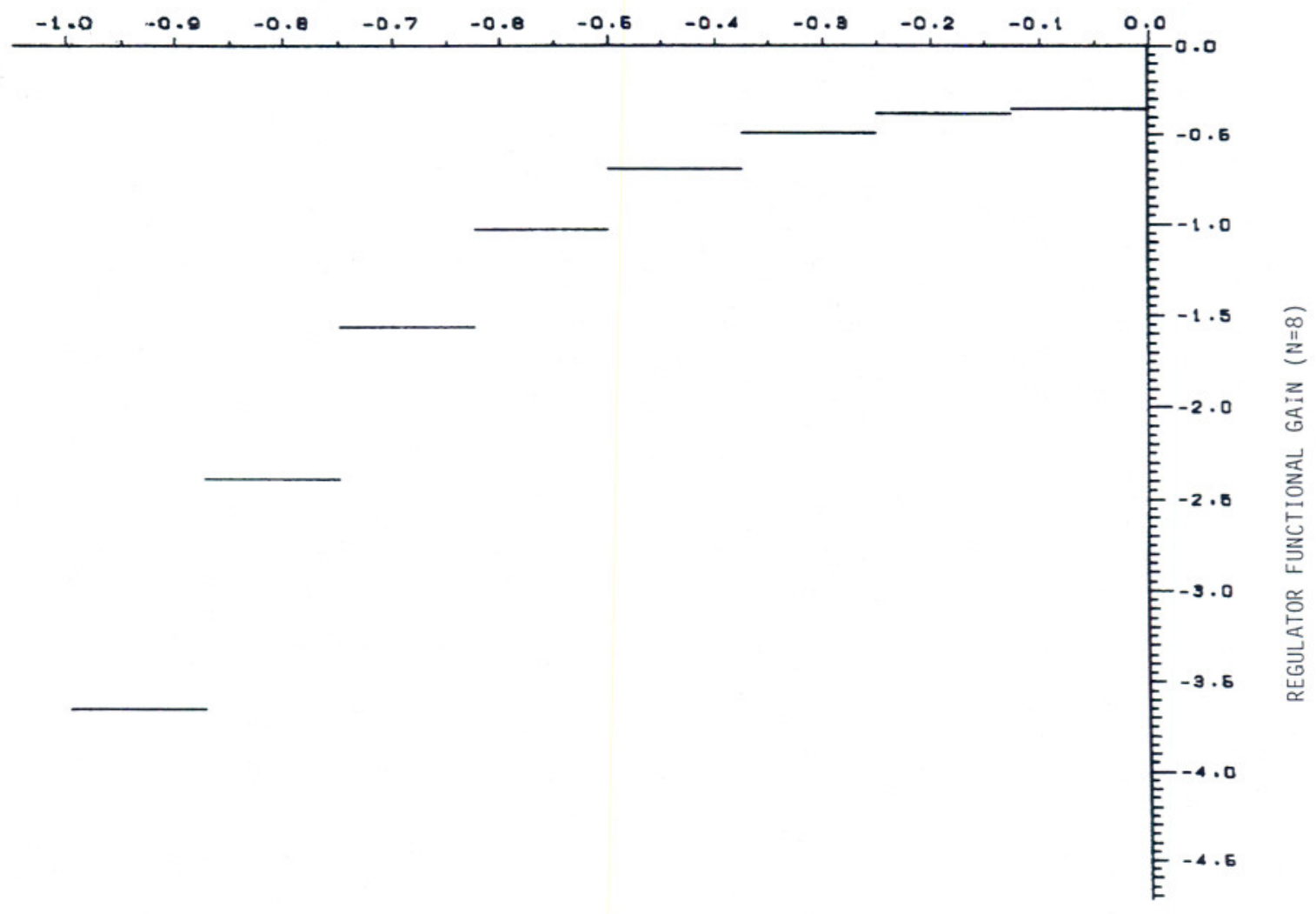

DELAY COORDINATE

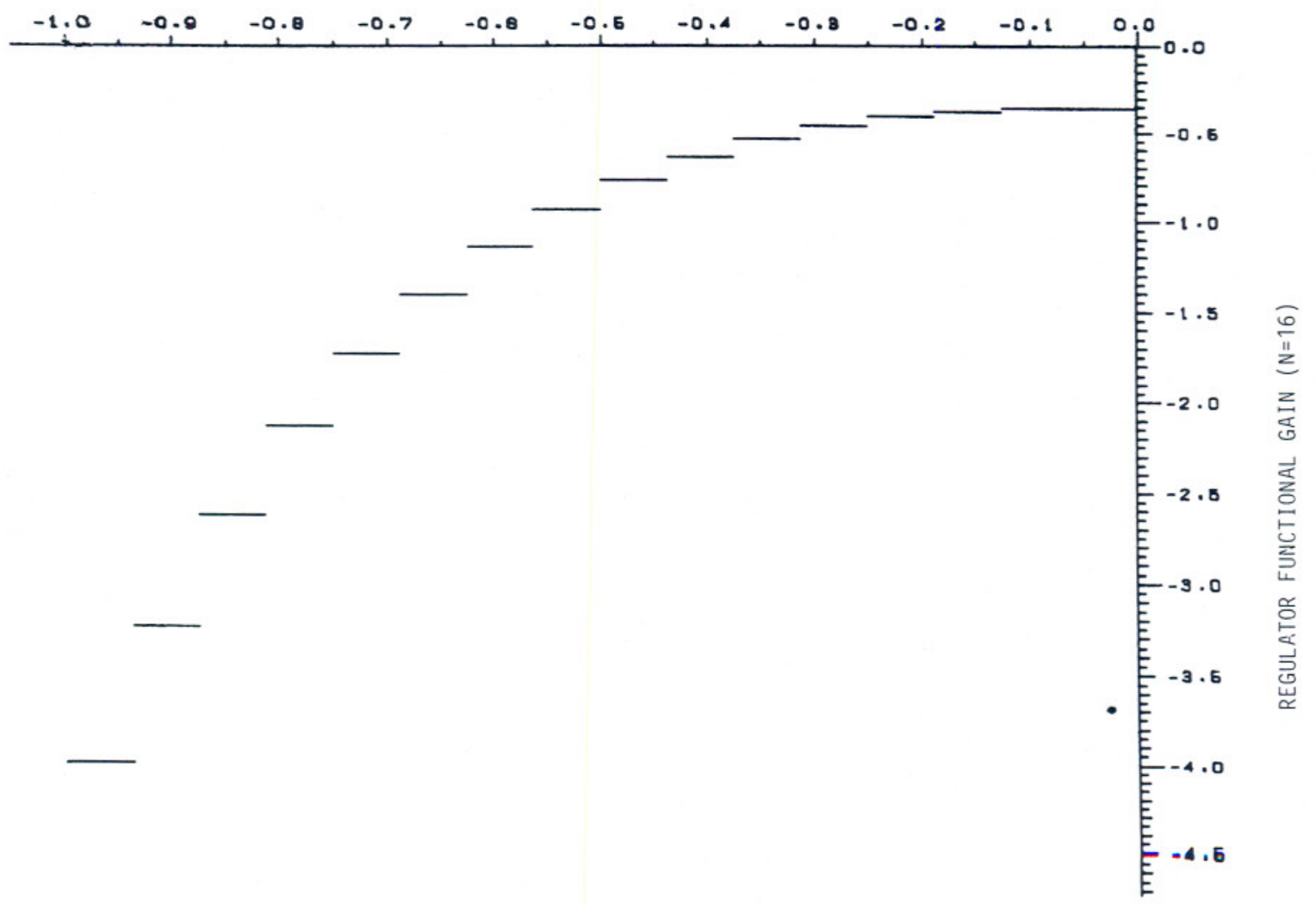


DELAY COORDINATE

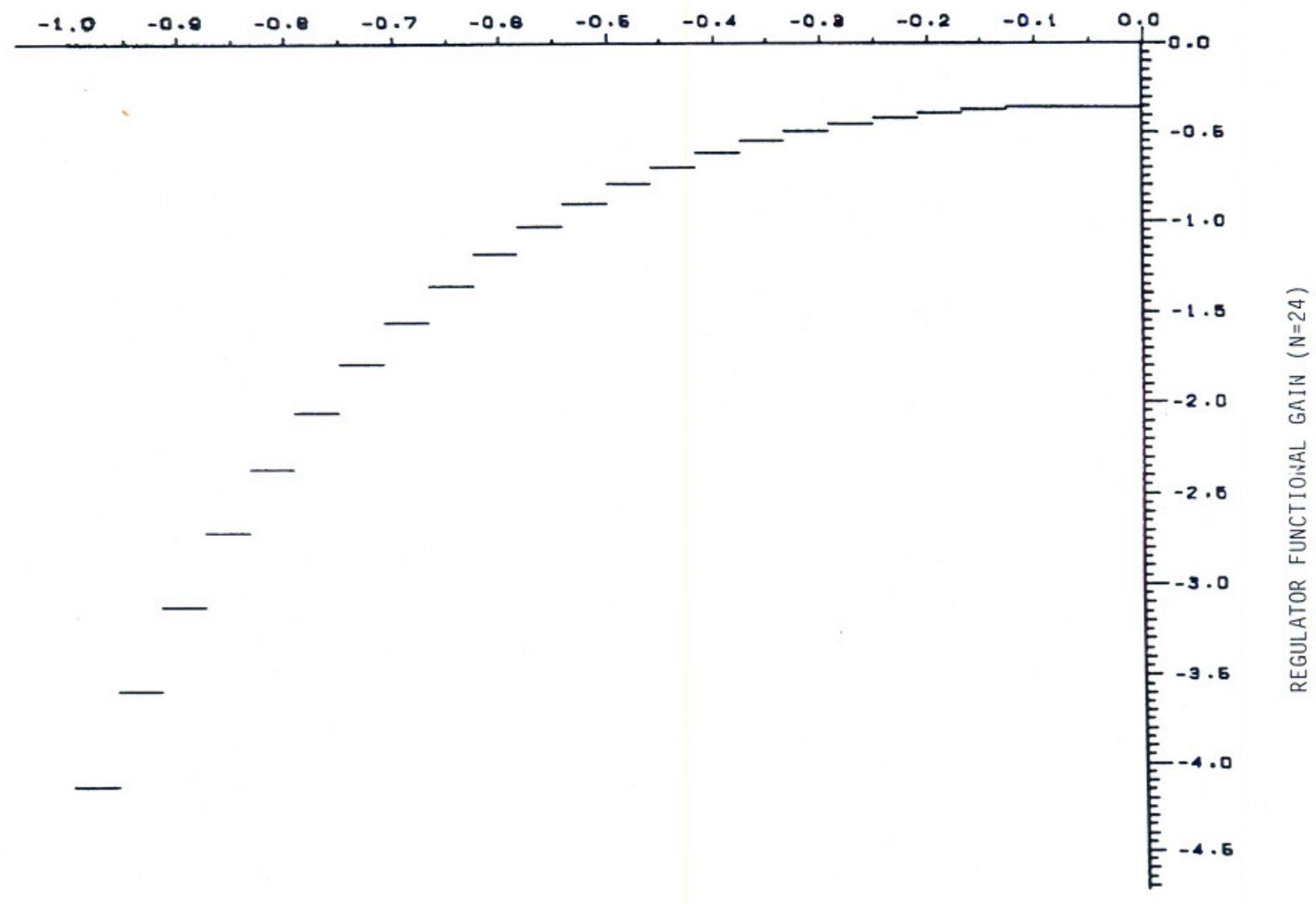

DELAY COORDINATE

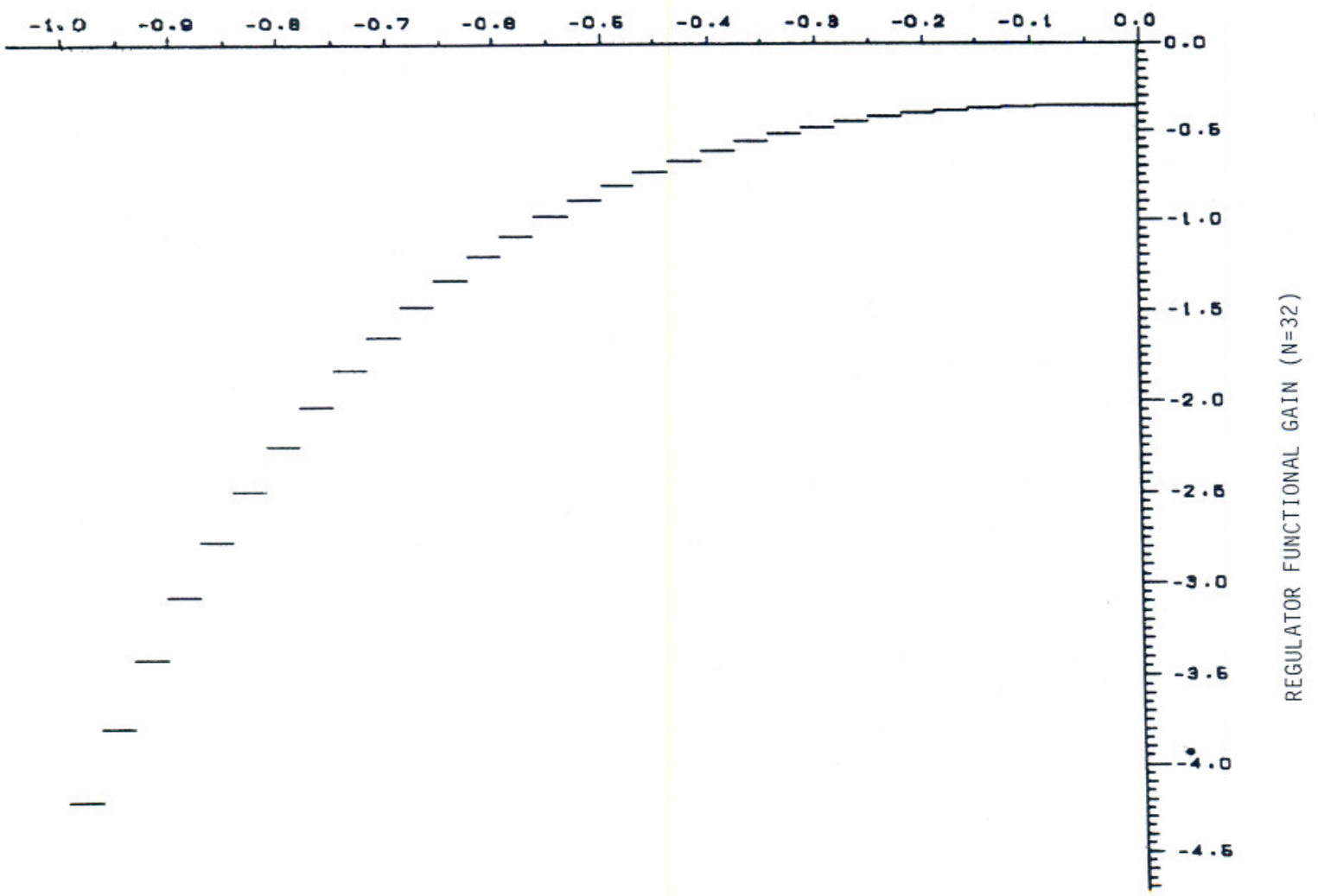

Figure 4. Hereditary system approximating optimal LQG functional control gains; $N=8,16,24,32$ 
Table IV. Hereditary system approximating optimal LQG scalar control gains

\begin{tabular}{ccccc}
\hline$N$ & 8 & 16 & 24 & 32 \\
\hline$\gamma_{0}^{N}$ & $-4 \cdot 4213$ & $-4 \cdot 4229$ & $-4 \cdot 4233$ & $-4 \cdot 4234$ \\
\hline
\end{tabular}

Table V. Hereditary system approximating optimal first-order compensator gains

\begin{tabular}{rllll}
\hline$N$ & $A_{\mathrm{c}}^{N}$ & $B_{\mathrm{c}}^{N} C_{\mathrm{c}}^{N}$ & \multicolumn{1}{c}{$J_{\mathrm{LQG}}^{N}$} & $J_{\mathrm{FO}}^{N}$ \\
\hline 8 & $-4 \cdot 835$ & $-16 \cdot 057$ & $1 \cdot 4042$ & $1 \cdot 5221$ \\
16 & $-4 \cdot 936$ & $-16 \cdot 343$ & $1 \cdot 403877$ & $1 \cdot 5298$ \\
24 & $-4 \cdot 959$ & $-16 \cdot 378$ & $1 \cdot 403856$ & $1 \cdot 5309$ \\
32 & $-4 \cdot 962$ & $-16 \cdot 404$ & $1 \cdot 403852$ & $1 \cdot 5317$ \\
\hline
\end{tabular}

is due to the nature of the input and output we have chosen and the usual duality which exists between the optimal regulator and filtering problems. The first 23 open-loop poles of the system $^{27}$ are given in Table 2. The approximating first-order compensator gains along with the corresponding and LQG closed-loop costs are given in Table 5. These costs were computed using an evaluation model obtained by setting $N=64$. Note that the performance of the firstorder controllers is within $10 \%$ of the performance of the LQG controllers. Once again, on the basis of the numerical results presented here, it appears that the approximating fixed-order compensator gains are converging as $N \rightarrow \infty$.

\section{SUMMARY AND CONCLUDING REMARKS}

We have proposed an approximation technique for computing optimal fixed-order compensators for distributed parameter systems. Our approach involves using the optimal projection theory for infinite-dimensional systems (which characterizes the optimal fixed-order compensator) developed in Reference 18 in conjunction with finite-dimensional approximation of the infinite-dimensional plant. We demonstrated the feasibility of our approach with two examples wherein we used spline-based Ritz-Galerkin finite element schemes to compute approximating optimal first-order controllers for one-dimensional, single-input/single-output parabolic (heat/diffusion) and hereditary control systems. The numerical studies that we have carried out indicate, at least for the examples that we have considered, that convergence of the compensator gains is achieved and that using the first-order controller would lead to only minimal performance degradation over a standard LQG compensator while simplifying the implementation significantly.

At this point one is led naturally to ask the question of whether or not a satisfactory convergence theory could be developed. We are working on this at present and expect that such a theory would conform closely in form and spirit to the convergence results for LQG approximation found in References 9 and 10 and outlined in Section 2 above. We also intend to consider our approximation ideas in the context of discrete-time or sampled data systems, and for continuous-time systems involving unbounded input and/or output (e.g. boundary control systems) and systems with control or measurement delays. ${ }^{11,12}$ Finally, we intend to investigate the application of our approximation framework to other infinite-dimensional 
control systems, in particular the vibration control of flexible structures (i.e. second-order systems such as wave, beam or plate equations).

\section{ACKNOWLEDGEMENTS}

The authors gratefully acknowledge Mr. S. W. Greeley, Mr. S. Richter and Mr. A. Daubendiek of Harris Corp. for carrying out the numerical computations reported in Section 3. We also wish to thank Ms. J. M. Straehla, also of Harris Corp., for preparing the original manuscript in TEX, and the reviewers for their helpful comments and suggestions.

D.S.B. was supported in part by the Air Force Office of Scientific Research under contracts F49620-86-C-0002 and F49620-89-C-0011. I.G.R. was supported in part by the Air Force Office of Scientific Research under grant AFOSR-87-0356. Part of this research was carried out while this author was a visiting scientist at the Institute for Computer Applications in Science and Engineering (ICASE) at the NASA Langley Research Center in Hampton, VA which is operated under NASA contracts NAS1-17070 and NAS1-18107.

\section{REFERENCES}

1. Banks, H. T. and J. A. Burns, 'Hereditary control problems: numerical methods based on averaging approximations', SIAM J. Control Optim., 16, 169-208 (1978).

2. Gibson, J. S., 'The Riccati integral equations for optimal control problems on Hilbert spaces', SIAM J. Control Optim., 17, 537-565 (1979).

3. Gibson, J. S., 'An analysis of optimal modal regulation: convergence and stability', SIAM J. Control Optim., 19, 686-707 (1981).

4. Kunisch, K., 'Approximation schemes for the linear quadratic optimal control problem associated with delay equations', SIAM J. Control Optim., 20, 506-540 (1982).

5. Gibson, J. S., 'Linear quadratic optimal control of hereditary differential systems: infinite dimensional Riccati equations and numerical approximations', SIAM J. Control Optim., 21, 95-139 (1983).

6. Banks, H. T., I. G. Rosen and K. Ito, 'A spline based technique for computing Riccati operators and feedback controls in regulator problems for delay equations', SIAM J. Sci. Stat. Comput., 5, 830-855 (1984).

7. Banks, H. T. and K. Kunisch, 'The linear regulator problem for parabolic systems', SIAM J. Control Optim., 22, 684-698 (1984).

8. Gibson, J. S. and I. G. Rosen, 'Numerical approximation for the infinite dimensional discrete time optimal linear quadratic regulator problem', SIAM J. Control Optim., 26, 428-451 (1988).

9. Gibson, J. S. and A. D. Adamian, 'Approximation theory for LQG optimal control of flexible structures', ICASE Report No. 88-48, Institute for Computer Applications in Science and Engineering, NASA Langley Research Center, Hampton, VA, August 1988.

10. Gibson, J. S. and I. G. Rosen, 'Computational methods for optimal linear quadratic compensators for infinite dimensional discrete time systems', Proc. 3rd Int. Conf. on Distributed Parameter Systems, Vorau, Austria, July 1986, Lecture Notes in Control and Information Sciences No. 102, Springer-Verlag, New York, 1987, pp. $120-135$.

11. Gibson, J. S. and I. G. Rosen, 'Approximation of discrete time LQG compensators for distributed systems with boundary input and unbounded measurement', Automatica, 24, 517-529 (1988).

12. Gibson, J. S. and I. G. Rosen, 'Approximation in discrete time boundary control of flexible structures', Proc. 26th IEEE Conf. on Decision and Control, Los Angeles, CA, December 1987, pp. 535-540.

13. Liu, Y. and B. D. O. Anderson, 'Controller reduction via stable factorization and balancing', Int. J. Control, 44, 507-531 (1986).

14. Hyland, D. C. and D. S. Bernstein, 'The optimal projection equations for fixed-order dynamic compensation', IEEE Trans. Automatic Control, AC-29, 1034-1037 (1984).

15. Johnson, T. L., 'Optimization of low order compensators for infinite dimensional systems', Proc. 9th IFIP Symp. on Optimization Techniques, Warsaw, September 1979, pp. 394-401.

16. Pearson, R. K., 'Optimal fixed form compensators for large space structures', in ACOSS Six (Active Control of Space Structures), RADC-TR-81-289, Rome Air Development Center, Griffiss Air Force Base, New York, 1981.

17. Johnson, T. L., 'Progress in modelling and control of flexible spacecraft', J. Franklin Inst., 315, 495-520 (1983).

18. Bernstein, D. S. and D. C. Hyland, 'The optimal projection equations for finite-dimensional fixed-order dynamic compensation of finite dimensional systems', SIAM J. Control Optim., 24, 122-151 (1986). 
19. Richter, S., 'A homotopy algorithm for solving the optimal projection equations for fixed order dynamic compensation: existence, convergence, and global optimality', Proc. Am. Control Conf., Minneapolis, MN, June 1987 , pp. $1527-1531$.

20. Greeley, S. W. and D. C. Hyland, 'Reduced order compensation: LQG reduction versus optimal projection using a homotopic continuation method', Proc. IEEE Conf. on Decision and Control, Los Angeles, CA, December 1987, pp. 742-747.

21. Curtain, R. F. and A. J. Pritchard, Infinite Dimensional Linear Systems Theory, Springer-Verlag, New York, 1978.

22. Balakrishnan, A. V., Applied Functional Analysis, Springer-Verlag New York, 1981.

23. Pazy, A., Semigroups of Linear Operators and Applications to Partial Differential Equations, Springer-Verlag, New York, 1983.

24. Smith, M. C., 'On minimal order stabilization of single loop plants', Syst. Control Lett., 7, 39-40 (1986).

25. Swartz, B. K. and R. S. Varga, 'Error bounds for spline and L-spine interpolation', J. Approx. Theory, 6, 6-49 (1972).

26. Ito, K. and F. Kappel, 'A uniformly differentiable approximation scheme for delay systems using splines', Technical Report 87-94, Institut für Mathematik, Technische Universität Graz and Universität Graz, Graz, Austria, 1987; Appl. Math. Optim., submitted.

27. Ito, K., 'On the approximation of eigenvalues associated with functional differential equations', J. Diff. Equations, 60, 285-300 (1985). 